\title{
Stress in an Infinite Medium around a Spheroidal Cavity Applied with Hydrostatic Pressure
}

\author{
by \\ T. Hirono \\ Meteorological Research Institute \\ and T. Usami \\ Central Meteorological Observatory
}

(Received March 22, 1954)

\begin{abstract}
Some of Japanese seismologists have been considering the effect of magma lying under the Earth's crust as the real origin of an earthquake, though there are some disagreements among them concerning what the effect of magma is: explosion or simple pressure. The authors have the opinion that the pressure of magma is to rise to break rocks surrounding its cavity and cause an earthquake, and theoretically investigate the relation between the shape of cavity and the breaking point around it. The two shapes, prolate and oblate spheroids, are dealt with, and it is assumed that one of these cavities is lying in an infinite elastic body, in which magma presses hydrostatically its boundary surface. The stress and then the deformation energy in medium around the cavity were calculated, and it is found that the points where the latter takes the maximum value were both poles for the prolate, and those on the equatorial circle for the oblate spheroid. These are considered to be the places to break at first according to von Mieses' theory. 'These considerations indicate that the points on a cavity with the smallest radius of curvature are liable to break first.
\end{abstract}

\section{Introduction}

In recent years, two leading theories concerning the mechanism of the earthquake have been prevailing among seismologists : the elastic rebound theory and the: magma intrusion theory. The rebound theory which describes the earthquake as the waves generated from the plane of an active fault as it dislocates, has been accepted by most seismologists nowadays. But it has been seldom discussed what agency could bring a fault into dislocation, though some of scholars attribute that vaguely to a shearing force occurring in such a vast land mass as Japan Proper as a whole.

In reference to this problem, Matuzawa [1] proposed lately an idea of the earthquake field under which he presumed a layer in some depth bounded in horizontal dimension and rocks therein are in the state of critical condition of melting. He thinks that its pressure rises higher in the process of rock-melting, and finally causes breaking in the medium surrounding the layer. At first, a major breaking is supposed to occur at a neighbourhood of the lateral boundary of the layer. Then minor breakings spread over to other regions neighbouring the molten lava layer. 
The magma intrusion theory which Isнiмото [2] brought forward states that an explosive high pressure, which is created suddenly in the magmatic rock as a result of its phase transition happening at some instant in its cooling process, breaks a weak point of the surrounding rocks. Then the rush of magma through a newly opened fissure causes magma to collide against the end wall of the fissure originating an earthquake tremor. TAKAGI's theory of earthquake mechanism [3] is similar to, but somewhat different from, that of Isнiмото. He insists that the earthquake is caused when a shock wave bursting from a small part within a magma cavity dashes against the cavity wall after travelling through some space in the cavity.

On the other hand, WADATI [4], studying Ishimoto's paper, expressed his opinion that earthquake tremor would be caused not by a magma collision but by a split of rock itself, though magma might act as an agency breaking the rocks.

Matuzawa's theory agrees with Wadati's in fundamental points. Further, Matuzawa did not consider magma to exist a priori, but to appear temporarily through the melting of rocks in the special layer mentioned above, which is destined to be cooled into solid rocks again. This cooling, he thinks, happens by increased heat conduction as a result of the breaking of rocks neighbouring the layer. He compared this whole process to a thermo-engine driven by intrinsic heat energy in the interior of the Earth.

The reason why Isнiмото could not employ the idea of the shattering of rocks as an origin of the earthquake seems to be that the distribution of initial motion of most shocks indicates at their foci the so-called cone type pull-push distribution with push sense inside of the cone. This fact seemed to be unexplainable by any breaking of rocks except by his theory [5]. Afterwards, however, another kind of cone type was discovered by Sakata [6] and Matuzawa [7], which undermined Ishimoto's theory; it has a pull sense inside of a cone.

Then the problem abandoned by IsHimoto comes out again, that is, how the cone type distribution could be possible if a shock is caused by a rock breaking by virtue of magma pressure. The authors have been trying to clarify this problem, and as a first step, how the rock breaking could occur by magma pressure will be studied here.

Matuzawa [1] has already investigated the rock breaking problem in connection with his earthquake field theory in the case of a surface layer with clamped circular boundary and with bottom surface pressed by hydrostatic pressure. The authors' calculation was performed without knowing his work, and their problem was found to be somewhat similar to his, but the treated cases have some interesting matters from other points of view.

The authors have the same opinion with WADATI on the earthquake mechanism. But they have no intention to present here any elaborate theory such as stated by Matuzawa. They want only to emphasize that the shape of magma cavity plays an important role in causing the breaking of the surrounding medium. And some examples with different shapes of cavity will be shown in this paper.

Now, a magma cavity is assumed to exist in such deep places that we can neglect the effect of the Earth's surface. The shape of cavity may be imagined to be something like lacolith or dyke which can be commonly observed in the fields. Further it ought to be remarked that the cavity must have at least one magmaconveying channel or fissure leading down to a vast reservoir of magma or of cri- 
tically heated rock on such a scale as batholith observable in the fields. This underlying reservoir may correspond to Matuzawa's thermo-engine.

Then the authors' problem is to find where the breaking could happen in the medium around the cavity as the magma pressure increases by some cause or other. It may be obvious that the breaking place and the running direction of fissure produced depend on the shape of cavity. The shapes here employed for investigation are prolate and oblate spheroids. Although these can not be considered to represent all kinds of cavities, the nature revealed in these cases would suggest something in common. In addition, in our theory, the effect of the vent conveying magma from an underlying region is neglected. The cavity wall is assumed to be everywhere subject to an equal hydrostatic pressure of magma and the state in question is that before breaking. Then the resultant stress in the medium surrounding the cavity is evaluated by the theory of elasticity. As for the theory of breaking, Mohr's maximum shear force theory and von Mieses' maximum deformation energy theory are well known, and the latter is employed in this paper.

\section{Part I. Cavity of Prolate Spheroid}

\section{Equations of equilibrium and their solutions}

The equations of equilibrium of elastic bodies in spheroidal coordinates $(\xi, \eta$, $\varphi)$ are

$$
\left\{\begin{array}{l}
(\lambda+2 \mu) h_{1} \frac{\partial \Delta}{\partial \xi}-2 \mu h_{2} h_{3} \frac{\partial}{\partial \eta}\left(\frac{\pi}{h_{3}}\right)=0 \\
(\lambda+2 \mu) h_{3} \frac{\partial \Delta}{\partial \eta}+2 \mu h_{1} h_{3} \frac{\partial}{\partial \xi}\left(\frac{\pi}{h_{3}}\right)=0
\end{array}\right.
$$

where $\lambda$ and $\mu$ are Lame's elastic constants. The equation with respect to the $\varphi$ component is omitted because of the nature under discussion being symmetry con. cerning the axis of revolution. The relation between spheroidal coordinates and Cartesian coordinates in the case of prolate spheroid is

$$
z+i \sqrt{x^{2}+y^{2}}=c \cosh (\xi+i \eta),
$$

or

$$
\frac{x^{2}+y^{2}}{c^{2}}+\frac{z^{2}}{\sinh ^{2} \xi}+\frac{z^{2}}{c^{2} \cosh ^{2} \xi}=1, \quad \frac{x^{2}+y^{2}}{c^{2} \cos ^{2} \eta}-1
$$

where the $z$ axis coincides with that of revolution. Further, $u$ and $v$ are compo. nents of displacement referred to $\xi$ and $\eta$, and

$$
\begin{gathered}
\Delta=h_{1} h_{2} h_{3}\left\{\frac{\partial}{\partial \xi}\left(\frac{u}{h_{2} h_{3}}\right)+\frac{\partial}{\partial \eta}\left(\frac{v}{h_{1} h_{3}}\right)\right\} \\
2 \pi=h_{1} h_{2}\left\{\frac{\partial}{\partial \xi}\left(\frac{v}{h_{2}}\right)-\frac{\partial}{\partial \eta}\left(\frac{u}{h_{1}}\right)\right\} \\
\left\{\begin{array}{l}
\frac{1}{h^{2}{ }_{1}}=\left(\frac{\partial x}{\partial \xi}\right)^{2}+\left(\frac{\partial y}{\partial \xi}\right)^{2}+\left(\frac{\partial z}{\partial \xi}\right)^{2}=c^{2}\left(\cosh ^{2} \xi-\cos ^{2} \eta\right)=\frac{1}{h_{2}^{2}} \equiv \frac{1}{h^{2}} \\
\frac{1}{h^{2}{ }_{3}}=\left(\frac{\partial x}{\partial \varphi}\right)^{2}+\left(\frac{\partial y}{\partial \varphi}\right)^{2}+\left(\frac{\partial z}{\partial \mathscr{p}}\right)^{2}=c^{2} \sinh ^{2} \xi \sin ^{2} \eta
\end{array}\right.
\end{gathered}
$$


If $\widehat{\xi \xi}, \widehat{\eta \eta}, \widehat{\rho \varphi}, \widehat{\xi \eta}, \widehat{\eta \varphi}$ and $\widehat{\xi \varphi}$ denote the six stress components, they are expressed :as follows,

(6)

$$
\begin{aligned}
& \widehat{\xi \xi}=\lambda \Delta+2 \mu\left(h_{1} \frac{\partial u}{\partial \xi}-v \frac{\partial h_{1}}{\partial \eta}\right) \\
& \widehat{\eta \eta}=\lambda \Delta+2 \mu\left(h_{1} \frac{\partial v}{\partial \eta}-u \frac{\partial h_{1}}{\partial \xi}\right) \\
& \widehat{\rho \varphi}=\lambda \Delta+2 \mu\left(-u \frac{h_{1}}{h_{3}} \partial h_{3}-v \frac{h_{1}}{h_{3}} \frac{\partial h_{3}}{\partial \eta}\right) \\
& \widehat{\xi \eta}=\mu\left\{\frac{\partial}{\partial \xi}\left(v h_{1}\right)+\frac{\partial}{\partial \eta}\left(u h_{1}\right)\right\} \\
& \widehat{\xi \varphi}=\widehat{\eta \varphi}=0 .
\end{aligned}
$$

Now the cavity is assumed to be a prolate spheroid expressed by $\xi=\xi_{0}$, and on which surface constant hydrostatic pressure operates. Then the boundary con,ditions are given by

$$
\left.\begin{array}{l}
\widehat{\xi \xi}=-A \\
\widehat{\xi \eta}=0
\end{array}\right\} \quad \text { at } \xi=\xi_{0},
$$

where $A$ means the given hydrostatic pressure.

From (1), (3), (4) and (5), we get

$$
\left\{\begin{array}{l}
\frac{\partial^{2} \Delta}{\partial \xi^{2}}+\operatorname{coth} \xi \frac{\partial \Delta}{\partial \xi}+\frac{\partial^{2} \Delta}{\partial \eta^{2}}+\cot \eta \frac{\partial \Delta}{\partial \eta}=0 \\
\frac{\partial^{2} \pi}{\partial \xi^{2}}+\operatorname{coth} \xi \frac{\partial \pi}{\partial \xi}-\frac{\pi}{\sinh ^{2} \xi}+\frac{\partial^{2} \pi}{\partial \eta^{2}}+\cot \eta \frac{\partial \pi}{\partial \eta}-\frac{\pi}{\sin ^{2} \eta}=0 .
\end{array}\right.
$$

Writing

$$
\Delta=G(\xi) H(\eta), \quad \pi=G^{\prime}(\xi) H^{\prime}(\eta),
$$

we can decompose (8) into

$$
\begin{gathered}
\left\{\begin{array}{c}
\frac{d^{2} G}{d \xi^{2}}+\operatorname{coth} \xi \frac{d G}{d \xi}-A_{n} G=0 \\
\frac{d^{2} H}{d \eta^{2}}+\cot \eta \frac{d H}{d \eta}+A_{n} H=0,
\end{array}\right. \\
\left\{\begin{array}{l}
\frac{d^{2} G^{\prime}}{d \xi^{2}}+\operatorname{coth} \xi \frac{d G^{\prime}}{d \xi}-\left(\begin{array}{c}
1 \\
\sinh ^{2} \xi
\end{array}+A_{n^{\prime}}{ }^{\prime}\right) G^{\prime}=0 \\
\frac{d^{2} H^{\prime}}{d \eta^{2}}+\cot \eta \frac{d H^{\prime}}{d \eta}-\left(\frac{1}{\sin ^{2} \eta}-A_{n}^{\prime}\right) H^{\prime}=0,
\end{array}\right.
\end{gathered}
$$

'where $A$ and $A^{\prime}$ are arbitrary constants, and if we put, referring to Hobson* p. .413,

$$
A_{n}=A_{n}{ }^{\prime}=n(n+1),
$$

particular solution of $G$ may be given by a sphericas function of either $P_{n}(\cosh \xi)$ or $Q_{n}(\cosh \xi)$, and $H$, by either $P_{n}(\cos \eta)$ or $Q_{n}(\cos \eta)$, while $G^{\prime}$ is given by either

* Hobson's "The Theory of Spherical and Ellipsoidal Harmonics" (1931) will be cited frequently below as simply "Hobson". 
$P_{n}{ }^{1}(\cosh \xi)$ or $Q_{n}{ }^{1}(\cosh \xi)$, and $H^{\prime}$, by either $P_{n}{ }^{1}(\cos \eta)$ or $Q_{n}{ }^{1}(\cos \eta)$ for $n \geqq 1$.

The relations between $P_{n}$ and $P_{n}{ }^{1}$, and $Q_{n}$ and $Q_{n}{ }^{1}$ are given by

$$
\left\{\begin{aligned}
P_{n}{ }^{1}(x) & =\left(1-x^{2}\right)^{1 / 2} \frac{d P_{n}}{d x}, & & x \leqq 1 . \\
Q_{n}{ }^{1}(x) & =\left(x^{2}-1\right)^{1 / 2} \frac{d Q_{n}}{d x}, & & x \geqq 1 .
\end{aligned}\right.
$$

Among these solutions those suitable to our problem are $P_{n}(\cos \eta), Q_{n}(\cosh \xi)$, $P_{n}{ }^{1}(\cos \eta)$ and $Q_{n}{ }^{1}(\cosh \xi)$, hence we have

$$
\left\{\begin{aligned}
\Delta_{n} & =B_{n} P_{n}(\cos \eta) Q_{n}(\cosh \xi) \\
\pi_{n} & =B_{n}{ }^{\prime} P_{n}{ }^{1}(\cos \eta) Q_{n}{ }^{1}(\cosh \xi),
\end{aligned}\right.
$$

where $B_{n}$ and $B_{n}^{\prime}$ are constants, and by introducing these into (1), we may find' them related to each other by

$$
\left\{\begin{array}{l}
B_{n}=\frac{2 \mu}{\lambda+2 \mu} n(n+1) B_{n}{ }^{\prime}, . \quad n \geqq 1 \\
B_{0}=B_{0}{ }^{\prime}=0 .
\end{array}\right.
$$

Now putting

$$
\frac{u}{h}=U, \quad \frac{v}{h}=V,
$$

and rewriting (3) and (4), we get

$$
\left\{\begin{aligned}
-\frac{\Delta}{h^{2}} & =\frac{\partial U}{\partial \xi}+\operatorname{coth} \xi \cdot U+\frac{\partial V}{\partial \eta}+\cot \eta \cdot V \\
\frac{2 \pi}{h^{2}} & =\frac{\partial V}{\partial \xi}-\frac{\partial U}{\partial \eta} .
\end{aligned}\right.
$$

Cancelling $V$ from (16), we have

(17) $\frac{\partial^{2} U}{\partial \xi^{2}}+\operatorname{coth} \xi \frac{\partial U}{\partial \xi}-\frac{1}{\sinh ^{2} \xi} U+\cot \eta \frac{\partial U}{\partial \eta}=\frac{\partial}{\partial \xi}\left(\begin{array}{c}\Delta \\ h^{2}\end{array}\right)-\frac{\partial}{\partial \eta}\left(\begin{array}{c}2 \pi \\ h^{2}\end{array}\right)-\cot \eta \frac{2 \pi}{h^{2}}$, or by (13),

$$
\begin{aligned}
=B_{n} & P_{n}\left\{Q_{n}{ }^{1}\left(\cosh ^{2} \xi-\cos ^{2} \eta\right)+2 Q_{n} \cosh \xi \sinh \xi\right\} c^{2} \\
& -2 B_{n}{ }^{\prime} Q_{n}{ }^{1}\left\{n(n+1)\left(\cosh ^{2} \xi-\cos ^{2} \xi\right) P_{n}+2 P_{n}{ }^{1} \cos \eta \sin \eta\right\} c^{2},
\end{aligned}
$$

Similarly, cancelling $U$ from (16), we get

$$
\begin{aligned}
& \frac{\partial^{2} V}{\partial \xi^{2}}+\operatorname{coth} \xi \frac{\partial V}{\partial \xi}+\frac{\partial^{2} V}{\partial \eta^{2}}+\cot \eta \frac{\partial V}{\partial \eta}-\frac{1}{\sin ^{2} \eta} V=\frac{\partial}{\partial \eta}\left(\frac{\Delta}{h^{2}}\right)+\frac{\partial}{\partial \xi}\left(\frac{2 \pi}{h^{2}}\right) \\
& \quad+\operatorname{coth} \xi \cdot \frac{2 \pi}{h^{2}}=-B_{n} Q_{n}\left\{P_{n}{ }^{1}\left(\cosh ^{2} \xi-\cos ^{2} \eta\right)-2 P_{n} \sin \eta \cos \eta\right\} c^{2} \\
& \quad+2 B_{n}{ }^{\prime} P_{n}{ }^{1}\left\{n(n+1)\left(\cosh ^{2} \xi-\cos ^{2} \eta\right) Q_{n}+2 Q_{n}{ }^{1} \cosh \xi \sinh \xi\right\} c^{2} .
\end{aligned}
$$

The solutions of (17) and (18) are

$$
\left\{\begin{aligned}
U_{n} / c^{2} & =\left(c_{1} \cosh ^{2} \xi \cdot Q_{n}{ }^{1}+c_{2} \sinh \xi \cosh \xi \cdot Q_{n}\right) P_{n}-c_{1} Q_{n}{ }^{1} P_{n} \cos ^{2} \eta \\
& +c_{4} Q_{n}{ }^{1} P_{n}{ }^{1} \cos \eta \sin \eta \\
V_{n} / c^{2} & =\left(c_{1} \cosh ^{2} \xi \cdot Q_{n}+c_{4} \cosh { }_{2}^{1} \xi \sinh \xi \cdot Q_{n}{ }^{1}\right) P_{n}{ }^{1}-c_{1} Q_{n} P_{n}{ }^{1} \cos ^{2} \eta \\
& -c_{2} Q_{n} P_{n} \cos \eta \sin \eta
\end{aligned}\right.
$$


where

$$
\begin{gathered}
c_{1}=\frac{B_{n}+6 B_{n}{ }^{\prime} n(n+1)}{8 n(n+1)-6}, \quad c_{2}=\frac{\{n(n+1)-1\} B_{n}-2 n^{2}(n+1)^{2} B_{n}{ }^{\prime}}{4 n(n+1)-3}, \\
c_{4}=\frac{-B_{n}+2\{n(n+1)-3\} B_{n^{\prime}}{ }^{\prime}}{4 n(n+1)-3} .
\end{gathered}
$$

$U$ and $V$ here obtained are the particular solutions corresponding to $\Delta_{n}$ and $\widetilde{\pi}_{n}$ given by (13), while the complementary solutions of $U$ and $V$ are easily obtained by putting $\Delta$ and $\widetilde{v}$ equal to zero in (17) and (18), thus,

$$
\begin{cases}U_{0} / c^{2}=D_{0} / \sinh \xi, & V_{0} / c^{2}=0 \\ U_{n} / c^{2}=D_{n} P_{n} Q_{n}^{1}, & V_{n} / c^{2}=-D_{n} P_{n}^{1} Q_{n} .\end{cases}
$$

By summing up these solutions by $n$, we may get the general solution. As the matter now under consideration is of the nature of symmetry about the $z$-axis, only an even number may be taken for $n$.

\section{The boundary condition (1)}

The boundary condition to be satisfied by $\widehat{\xi \xi}$ can be written, referring to (6) and (7), thus,

$$
-A=\lambda \Delta+2 \mu\left\{\left(h^{2} \frac{\partial U}{\partial \xi}+\frac{U}{2}-\frac{\partial h^{2}}{\partial \xi}\right)-\frac{V}{2} \frac{\partial h^{2}}{\partial \eta}\right\}, \quad \text { at } \xi=\xi_{0} .
$$

Introducing $h$ of (5) in this, we have,

$$
\begin{aligned}
-c^{2}\left(\cosh ^{2} \xi\right. & \left.-\cos ^{2} \eta\right)^{2} A=c^{2} \lambda\left(\cosh ^{2} \xi-\cos ^{2} \eta\right)^{2} \Delta \\
& +2 \mu\left\{\left(\cosh ^{2} \xi-\cos ^{2} \eta\right) \frac{\partial U}{\partial \xi}-\sinh \xi \cosh \xi \cdot U+\cos \eta \sin \eta \cdot V\right\},
\end{aligned}
$$

where $\xi$ are placed for $\xi_{0}$ for brevity's sake.

At first, let us put only the particular solutions (19) into $U$ and $V$ in the above equation. Then, the right hand side of (23) takes the from

$$
\begin{aligned}
\sum_{n}\left(c_{2}\right. & \left.+c_{5}\right)\left(\cosh ^{2} \xi-\cos ^{2} \eta\right) P_{n} Q_{n}+\sum_{n}\left(c_{1}+c_{2}\right) \sinh \xi \cosh \xi \cdot P_{n} Q_{n}{ }^{1} \\
& +\sum_{n} c_{1} P_{n} Q_{n}{ }^{\prime \prime}\left(\cosh ^{2} \xi-\cos ^{2} \eta\right)+\sum_{n} c_{4} P_{n}{ }^{1} Q_{n}{ }^{\prime \prime} \cos \eta \sin \eta \\
& +\sum_{n}^{1} c_{1} \cos \eta \sin \eta \cdot Q_{n} P_{n}{ }^{1}
\end{aligned}
$$

where factor

$$
2 \mu c^{2}\left(\cosh ^{2} \xi-\cos ^{2} \eta\right)
$$

is discarded for the present, and further we put

$$
c_{5}=\frac{\lambda B_{n}}{2 \mu}, \quad Q_{n}^{\prime \prime}=\frac{d^{2} Q_{n}}{d \xi^{2}} .
$$

Then, using the relation

$$
-P_{n}{ }^{1} \sin \eta=n \cos \eta \cdot P_{n}-n P_{n-1},
$$

we may write (24) as follows,

$$
\begin{aligned}
& \sum\left\{\left(c_{2}+c_{5}\right) \cosh ^{2} \xi \cdot Q_{n}+\left(c_{1}+c_{2}\right) \sinh \xi \cosh \xi \cdot Q_{n}{ }^{1}+c_{1} \cosh ^{2} \xi \cdot Q_{n}{ }^{\prime \prime}\right\} P_{n} \\
& \left.\quad+\sum\left\{-c_{5}-c_{2}-n c_{1}\right) Q_{n}+\left(-c_{1}-n c_{4}\right) Q_{n}{ }^{\prime \prime}\right\} P_{n} \cos ^{2} \eta \\
& \quad+\sum\left(n c_{4} Q_{n}{ }^{\prime \prime}+n c_{1} Q_{n}\right) P_{n-1} \cos \eta
\end{aligned}
$$


Further, by means of the relations

$$
\left\{\begin{array}{l}
P_{n} \cos ^{2} \eta=\alpha_{n+2} P_{n+2}+\alpha_{n} P_{n}+\alpha_{n-2} P_{n-2} \\
P_{n-1} \cos \eta=\beta_{n} P_{n}+\beta_{n-2} P_{n-2}
\end{array}\right.
$$

where

$$
\left\{\begin{aligned}
\alpha_{n+2} & =\frac{(n+1)(n+2)}{(2 n+1)(2 n+3)}, \quad \alpha_{n}=\frac{(n+1)^{2}}{(2 n+1)(2 n+3)}+\frac{n^{2}}{(2 n+1)(2 n-1)}, \\
\alpha_{n-2} & =\frac{n(n-1)}{(2 n+1)(2 n-1)} \\
\beta_{n} & =\frac{n}{2 n-1}, \quad \beta_{n-2}=\frac{n-1}{2 n-1},
\end{aligned}\right.
$$

we can transfrom (28) into

$$
\sum\left(d_{n-2} P_{n-2}+d_{n} P_{n}+d_{n+2} P_{n+2}\right)
$$

where

$$
\left\{\begin{aligned}
& d_{n-2}=-\alpha_{n-2}\left\{\left(c_{5}+c_{2}+n c_{1}\right) Q_{n}+\left(c_{1}+n c_{4}\right) Q_{n}{ }^{\prime \prime}\right\}+\beta_{n-2} n\left(c_{4} Q_{n}{ }^{\prime \prime}+c_{1} Q_{n}\right) \\
& d_{n}=\left(c_{2}+c_{5}\right) \cosh ^{2} \xi \cdot Q_{n}+\left(c_{1}+c_{2}\right) \sinh \xi \cosh \xi \cdot Q_{n}{ }^{1}+c_{1} \cosh ^{2} \xi \cdot Q_{n}{ }^{\prime \prime} \\
&-\alpha_{n}\left\{\left(c_{5}+c_{2}+n c_{1}\right) Q_{n}+\left(c_{1}+n c_{4}\right) Q_{n}{ }^{\prime \prime}\right\}+\beta_{n} n\left(c_{4} Q_{n}{ }^{\prime \prime}+c_{1} Q_{n}\right) \\
& d_{n+2}=-\alpha_{n+2}\left\{\left(c_{5}+c_{2}+n c_{1}\right) Q_{n}+\left(c_{1}+n c_{4}\right) Q_{n}{ }^{\prime \prime}\right\} .
\end{aligned}\right.
$$

Next, let us substitute complementary solutions given by (21) into $U$ and $V$ in: (23). Then the right hand side of (23) is

$$
\begin{gathered}
2 \mu c^{2}\left(\cosh ^{2} \xi-\cos ^{2} \eta\right) \sum_{n}\left\{\frac{1}{c^{2}} \frac{\partial U_{n}}{\partial \xi}-\left(\sinh \xi \cosh \xi \cdot P_{n} Q_{n}{ }^{1}\right.\right. \\
\left.\left.+\cos \eta \sin \eta \cdot P_{n}{ }^{1} Q_{n}\right) \cdots \frac{D_{n}}{\cosh ^{2} \xi-\cos ^{2} \eta}\right\},
\end{gathered}
$$

where the second term in \{\} can be expanded by $P_{m}$, thus,

$$
\begin{aligned}
& \frac{\sinh \xi \cosh \xi \cdot P_{n} Q_{n}{ }^{1}+\cos \eta \sin \eta \cdot P_{n}{ }^{1} Q_{n}}{\cosh ^{2} \xi-\cos ^{2} \eta} \\
&=\sum_{m=0}^{\infty}\left(m+\frac{1}{2}\right) P_{m} \int_{-1}^{1} P_{m} \frac{\sinh \xi \cosh \xi \cdot P_{n} Q_{n}{ }^{1}+\cos \eta \sin \eta P_{n}{ }^{1} Q_{n}}{\cosh ^{2} \xi-\cos ^{2} \eta} d(\cos \eta) .
\end{aligned}
$$

Now, let us proceed to evaluate the integration involved here.

In Hobson p. 32 and p. 33. we find

$$
\left\{\begin{array}{l}
\left(\mu^{2}-1\right) \frac{d P_{n}}{d \mu}=n\left(\mu P_{n}-P_{n-1}\right) \\
\left(\mu^{2}-1\right) \frac{d P_{n}}{d \mu}=-(n+1)\left(\mu P_{n}-P_{n+1}\right),
\end{array}\right.
$$

where $\mu=\cos \eta$, and similar relations also hold for $Q_{n}$. From these, it follows that

(35) $\cosh \xi \sinh \xi \cdot Q_{n}^{2} P_{n}+\cos \eta \sin \eta \cdot Q_{n} P_{n}=n\left(\lambda^{2}-\mu^{2}\right) P_{n} Q_{n}-n\left(\lambda P_{n} Q_{n-1}-\mu P_{n-1} Q_{n}\right)$

$$
=-(n+1)\left(\lambda^{2}-\mu^{2}\right) P_{n} Q_{n}+(n+1)\left(\lambda P_{n} Q_{n+1}-\mu P_{n+1} Q_{n}\right) \text {, }
$$

where $\lambda=\cosh \xi$,

Again from Hobson p. 32, we have 
(36)

$$
\left\{\begin{array}{l}
(2 n-1) \mu P_{n-1}=n P_{n}+(n-1) P_{n-2} \\
(2 n-1) \lambda Q_{n-1}=n Q_{n}+(n-1) Q_{n-2}
\end{array}\right.
$$

Combining these, we have the equations

$$
\left\{\begin{array}{l}
(2 n-1)\left\{\mu P_{n-1} Q_{n}-\lambda P_{n} Q_{n-1}\right\}=(n-1)\left(P_{n-2} Q_{n}-P_{n} Q_{n-2}\right) \\
(2 n+3)\left\{\mu P_{n+1} Q_{n}-\lambda P_{n} Q_{n+1}\right\}=(n+2)\left(P_{n+2} Q_{n}-P_{n} Q_{n+2}\right) .
\end{array}\right.
$$

Introducing these into (35), we have

$$
\begin{aligned}
\cosh \xi \sinh \xi Q_{n}{ }^{1} P_{n} & +\cos \eta \sin \eta \cdot Q_{n} P_{n}{ }^{1}=n\left(\lambda^{2}-\mu^{2}\right) P_{n} Q_{n} \\
& +\frac{n(n-1)}{2 n-1}\left(P_{n-2} Q_{n}-P_{n} Q_{n-2}\right)=-(n+1)\left(\lambda^{2}-\mu^{2}\right) P_{n} Q_{n} \\
& -\frac{(n+1)(n+2)}{2 n+3}\left(P_{n+2} Q_{n} \cdots P_{n} Q_{n+2}\right) .
\end{aligned}
$$

Equating the two right hand side forms, we have

$$
\begin{gathered}
\frac{(n+1)(n+2)}{2 n+3}\left(P_{n} Q_{n+2}-P_{n+2} Q_{n}\right)=(2 n+1)\left(\lambda^{2}-\mu^{2}\right) P_{n} Q_{n} \\
+\frac{n(n-1)}{2 n-1}\left(P_{n-2} Q_{n}-P_{n} Q_{n-2}\right) .
\end{gathered}
$$

This is a kind of recurrence formula. Hence, using it repeatedly, we have

$(40)=\left\{(2 n+1) P_{n} Q_{n}+(2 n-3) P_{n-2} Q_{n-2}+\ldots \ldots+5 P_{2} Q_{2}\right\}\left(\lambda^{2}-\mu^{2}\right)+\frac{2}{3}\left(P_{0} Q_{2}-P_{2} Q_{0}\right)$.

Further, using the relations

$$
P_{0}=1, \quad P_{2}=\frac{1}{2}\left(3 \mu^{2}-1\right), \quad Q_{2}=P_{2}(\lambda) Q_{0}(\lambda)-\frac{3}{2} \lambda,
$$

from (38) and (40), the right hand side of (33) becomes

$$
\sum_{m}\left(m+\frac{1}{2}\right) P_{m} \int_{-1}^{1} P_{m}\left\{n P_{n} Q_{n}+\sum_{n=2,4,6}^{n}(2 n-3) P_{n-2} Q_{n-2}-\frac{\lambda}{\cosh ^{2} \xi-\cos ^{2} \eta}\right\} d \mu .
$$

For $m>n$, it becomes

$$
=\sum_{m=n+1}^{\infty}\left(m+\frac{1}{2}\right) P_{m} \int_{-1}^{1} \frac{-\lambda P_{m}}{\lambda^{2}-\mu^{2}} d \mu=\left\{\begin{array}{ll}
-\sum_{m=n+2}^{\infty}\left(m+\frac{1}{2}\right) 2 Q_{m} P_{m} & \text { for even } m \\
0 & \text { for odd } m
\end{array}\right\},
$$

because

$$
\int_{-1}^{1} P_{m} P_{n} d \mu=0, \quad(m \neq n), \quad \text { and } \quad Q_{m}=\int_{-1}^{1} \frac{P_{m} d \mu}{\lambda-\mu} .
$$

For $m=n$, it becomes

$(42 \mathrm{~b})=\left(m+\frac{1}{2}\right) P_{m}\left\{m \int_{-1}^{1}\left(P_{m}\right)^{2} Q_{m} d \mu-2 Q_{m}\right\}=-(m+1) Q_{m} P_{m}$, for even $m$.

And for $m<n$,

$$
=\sum_{m<n}\left(m+\frac{1}{2}\right) P_{m}\left\{(2 m+1) Q_{m} \int_{-1}^{1}\left(P_{m}\right)^{2} d \mu-2 Q_{m}\right\}=0 .
$$

Then, the form in \{ $\}$ of (32) may be reduced to

$$
\sum_{n} D_{n} Q_{n}{ }^{\prime \prime} P_{n}-\sum_{m} T_{m} P_{m}
$$


where

$$
T_{m}=-(2 m+1) Q_{m} \sum_{n=0}^{m-2} D_{n}-(m+1) Q_{m} D_{m}
$$

From (31) and (43), (23) may be reduced to

$$
\begin{aligned}
-\left(\cosh ^{2} \xi\right. & \left.-\frac{1}{3}\right) \frac{A}{2 \mu}+\frac{2}{3} \frac{A}{2 \mu} P_{2}=\sum_{n=2}^{\infty}\left(d_{n-2} P_{n-2}+d_{n} P_{n}+d_{n+2} P_{n+2}\right) \\
& +\sum_{n=0}^{\infty} D_{n} Q_{n}{ }^{\prime \prime} P_{n}-\sum_{m=0}^{\infty} T_{m} P_{m} .
\end{aligned}
$$

Equating each coefficient of $P_{n}$ in both sides, we have

$$
\left\{\begin{array}{l}
d_{n-2}+\left(D_{0} Q_{0}{ }^{\prime \prime}-T_{0}\right)=-\left(\cosh ^{2} \xi-\frac{1}{3}\right) \frac{A}{2 \mu} \\
d_{2}+d_{4-2}+\left(D_{2} Q_{2}^{\prime \prime}-T_{2}\right)=\frac{2}{3} \frac{A}{2 \mu} \\
d_{n+2}+d_{(n+2)}+d_{(n+4)-2}+\left(D_{n+2} Q_{n+2}^{\prime \prime}-T_{n+2}\right)=0 .
\end{array}\right.
$$

This is an infinite simultaneous linear equation with respect to $B_{n}$ and $D_{n}$.

\section{The boundary condition (2)}

Now, Iet us consider the boundary condition satisfied by $\overline{\xi \eta}$. From (5), (6) and (15), we have

$$
\begin{gathered}
\hat{\xi \eta}=\mu\left\{\frac{\partial}{\partial \xi}\left(V h^{2}\right)+\frac{\partial}{\partial \eta}\left(U h^{2}\right)\right\}, \\
=\mu h\left\{\frac{\partial V}{\partial \xi}+\frac{\partial U}{\partial \eta}-2 c^{2} h^{2}(V \cosh \xi \sinh \xi+U \cos \eta \sin \eta)\right\} .
\end{gathered}
$$

Putting only particular solutions into $U$ and $V$ involved in (47a), we have

$$
\begin{aligned}
(\widehat{\xi \eta})_{p}= & c^{2} \mu h^{2} \sum_{n}\left[-c_{4} P_{n}{ }^{1} Q_{n}{ }^{1}\left(\cosh ^{2} \xi+\cos ^{2} \eta\right)\right. \\
& \left.+\left\{n(n+1) c_{4}-c_{2}\right\}\left(Q_{n}{ }^{1} P_{n} \cos \eta \sin \eta+P_{n}{ }^{1} Q_{n} \sinh \xi \cosh \xi\right)\right] .
\end{aligned}
$$

Hobson p. 108 shows

$$
(2 n+1) \mu P_{n}^{m}(\mu)-(n-m+1) P_{n+1}^{m}(\mu)-(n+m) P_{n-1}^{m}(\mu)=0 .
$$

For $m=1$, this becomes

$$
\mu P_{n}{ }^{1}=\gamma_{n+1} P_{n+1}^{1}+\gamma_{n-1} P_{n-1}^{1} .
$$

where

$$
\gamma_{n+1}=\frac{n}{2 n+1}, \quad \gamma_{n-1}=\frac{n+1}{2 n+1} .
$$

By means of (50), we have

$$
\mu^{2} P_{n}{ }^{1}=\delta_{n+2} P_{n+2}^{1}+\delta_{n} P_{n}{ }^{1}+\delta_{n-2} P_{n-2}^{1},
$$

where

$$
\begin{aligned}
& \delta_{n+2}=\frac{n(n+1)}{(2 n+1)(2 n+3)}, \quad \delta_{n}=\frac{n(n+2)}{(2 n+1)(2 n+3)}+\frac{(n+1)(n-1)}{(2 n+1)(2 n-1)}, \\
& \delta_{n-2}=\frac{n(n+1)}{(2 n+1)(2 n-1)}
\end{aligned}
$$


On the other hand, from the relation

$$
P_{n+1}^{1}-P_{n-1}^{1}=(2 n+1) P_{n} \sin \eta .
$$

we have

$$
P_{n} \sin \eta \cos \eta=\varepsilon_{n+2} P_{n+2}^{1}+\varepsilon_{n} P_{n}{ }^{1}+\varepsilon_{n-2} P_{n-2}^{1},
$$

where

$$
\begin{aligned}
& \varepsilon_{n+2}=\frac{n+1}{(2 n+1)(2 n+3)}, \quad \varepsilon_{n}=\frac{n+2}{(2 n+1)(2 n+3)}-\frac{n-1}{(2 n+1)(2 n-1)}, \\
& \varepsilon_{n-2}=\frac{-n}{(2 n-1)(2 n+1)} .
\end{aligned}
$$

Introducing (52) and (55) into (48), we get

$$
(\widehat{\xi \eta})_{p}=c^{2} \mu h^{2} \sum_{n}\left\{e_{n+2} P_{n+2}^{1}+e_{n} P_{n}{ }^{1}+e_{n-2} P_{n-2}^{1}\right\},
$$

where

$$
\left\{\begin{aligned}
e_{n+2} & =-c_{4} Q_{n}{ }^{1} \delta_{n+2}+\left\{n(n+1) c_{4}-c_{2}\right\} Q_{n}{ }^{1} \varepsilon_{n+2} \\
e_{n}= & -c_{4} Q_{n}{ }^{1} \delta_{n}+\left\{n(n+1) c_{4}-c_{2}\right\} Q_{n}{ }^{1} \varepsilon_{n}-c_{4} Q_{n}{ }^{1} \cosh ^{2} \xi \\
& +\left\{n(n+1) c_{4}-c_{2}\right\} Q_{n} \sinh \xi \cosh \xi \\
e_{n-2}= & -c_{4} Q_{n}{ }^{1}{ }^{1}{ }{ }_{n-2}+\left\{n(n+1) c_{4}-c_{2}\right\} Q_{n}{ }^{1} \varepsilon_{n-2} .
\end{aligned}\right.
$$

Next, we will introduce the complementary solutions into $U$ and $V$ involved in (47a). Then we get

$$
\begin{aligned}
(\widehat{\xi \eta})_{c}= & \sum\left\{-2 D_{n} Q_{n}{ }^{1} P_{n}{ }^{1}+\frac{2 D_{n}}{\cosh ^{2} \xi-\cos ^{2} \eta}\left(P_{n}{ }^{1} Q_{n} \cosh \xi \sinh \xi\right.\right. \\
& \left.\left.-P_{n} Q_{n}{ }^{1} \cos \eta \sin \eta\right)\right\} c^{2} \mu n^{2},
\end{aligned}
$$

where the second term in \{ $\}$ can be expanded by $P_{m}{ }^{1}$, thus,

$$
\begin{aligned}
& \underline{P}_{0}{ }^{16} \quad \frac{\mathrm{h} \xi \sinh \xi-P_{n} Q_{n}{ }^{1} \cos \eta \sin \eta}{\cosh ^{2} \xi-\cos ^{2} \eta} \\
& \quad=\sum_{m=0}^{\infty} \cdot \frac{(2 m+1)}{2 m(m+1)} P_{m}{ }^{1} \int_{-1}^{1} P_{m}{ }^{1} \frac{P_{n}{ }^{1} Q_{n}}{\frac{\cosh \xi \sinh \xi-P_{n} Q_{n}{ }^{1} \cos \eta \sin \eta}{\cosh ^{2} \xi-\cos ^{2} \eta} d \mu .}
\end{aligned}
$$

Now, the evaluation of the integration involved will be considered below.

From Hobson p. 33,

$$
\left\{\begin{array}{l}
n P_{n}=\mu \frac{d P_{n}}{d \mu}-\frac{d P_{n-1}}{d \mu} \\
(n+1) P_{n}=-\mu \frac{d P_{n}}{d \mu}+\frac{d P_{n+1}}{d \mu} .
\end{array}\right.
$$

Forms similar to these hold for $Q_{n}$. From these, it follows that

$$
\begin{aligned}
& P_{n}{ }^{1} Q_{n} \cosh \xi \sinh \xi-P_{n} Q_{n}{ }^{1} \cos \eta \sin \eta=\frac{1}{n} P_{n}{ }^{1} Q_{n}{ }^{1}\left(\lambda^{2}-\mu^{2}\right) \\
& +\frac{1}{n}\left(\mu P_{n-1}{ }^{1} Q_{n}{ }^{1}-\lambda P_{n}{ }^{1} Q_{n-1}{ }^{1}\right)=\frac{-1}{n+1} P_{n}{ }^{1} Q_{n}{ }^{1}\left(\lambda^{2}-\mu^{2}\right)+\frac{1}{n+1}\left(\lambda Q_{n+1}{ }^{1} P_{n}{ }^{1}-\mu P^{1}{ }_{n+1} Q_{n}{ }^{1}\right) .
\end{aligned}
$$

Further, from Hobson p. 33, we have

$$
(2 n+1) \mu \frac{d P_{n}}{d \mu}=(n+1) \frac{d P_{n-1}}{d \mu}+n \frac{d P_{n+1}}{d \mu} .
$$


A form similar to this also holds for $Q_{n}$, and from these, it follows that

$$
\left\{\begin{array}{l}
(2 n-1)\left(\mu P_{n-1}^{1} Q_{n}{ }^{1}-\lambda P_{n}{ }^{1} Q^{1}{ }_{n-1}\right)=n\left(P_{n-2}^{1} Q_{n}{ }^{1}-P_{n}{ }^{1} Q^{1}{ }_{n-2}\right) \\
(2 n+3)\left(\mu P_{n+1}^{1} Q_{n}{ }^{1}-\lambda P_{n}{ }^{1} Q^{1}{ }_{n+1}\right)=(n+2)\left(P^{1}{ }_{n+2} Q_{n}{ }^{1}-P_{n}{ }^{1} Q^{1}{ }_{n+2}\right)
\end{array}\right.
$$

Putting these into (62), we get

$$
\begin{aligned}
P_{n}{ }^{1} Q_{n} \cosh \xi & \sinh \xi-P_{n} Q_{n}{ }^{1} \cos \eta \sin \eta=\frac{1}{n} P_{n}{ }^{1} Q_{n}{ }^{1}\left(\lambda^{2}-\mu^{2}\right) \\
& +\frac{1}{2 n-1}\left(P^{1}{ }_{n-2} Q_{n}{ }^{1}-P_{n}{ }^{1} Q^{1}{ }_{n-2}\right)=-\frac{1}{n+1} P_{n}{ }^{1} Q_{n}{ }^{1}\left(\lambda^{2}-\mu^{2}\right) \\
& +\frac{1}{2 n+3}\left(P_{n}{ }^{1} Q_{n+2}{ }^{1}-P_{n+2}{ }^{1} Q_{n}{ }^{1}\right) .
\end{aligned}
$$

By equating two forms in the right hand side of (65), we get a recurrence formula, that is,

$$
\frac{1}{2 n+3}\left(P_{n}{ }^{1} Q^{1}{ }_{n+2}-P^{1}{ }_{n+2} Q_{n}{ }^{1}\right)=\left(\frac{1}{n}+\frac{1}{n+1}\right) P_{n}{ }^{1} Q_{n}{ }^{1}\left(\lambda^{2}-\mu^{2}\right)+\frac{1}{2 n-1}\left(P_{n-2} Q_{n}{ }^{1}-P_{n}{ }^{1} Q_{n-2}{ }^{1}\right) .
$$

Then, using this relation repeatedly, we have

(66) $\frac{1}{2 n+3}\left(P_{n}{ }^{1} Q^{1}{ }_{n+2}-P^{1}{ }_{n+2} Q_{n}{ }^{1}\right)=\sum_{2}^{n}\left(\frac{1}{n}+\frac{1}{n+1}\right) P_{n}{ }^{1} Q_{n}{ }^{1}\left(\lambda^{2}-\mu^{2}\right)+\frac{1}{3}\left(P_{0}{ }^{1} Q_{2}{ }^{1}-P_{2}{ }^{1} Q_{0}{ }^{1}\right)$ 。 Taking into consideration

$$
P_{0}{ }^{1}=0, \quad P_{2}^{1}=3 \sin \eta \cos \eta,
$$

from (65), (66), we can transform the right hand side of (60) into

$$
\sum_{m=0}^{\infty} \frac{(2 m+1)}{2 m(m+1)} P_{m}{ }^{1} \int_{-1}^{1} P_{m}{ }^{1}\left\{\frac{1}{n} P_{n}{ }^{1} Q_{n}{ }^{1}+\sum_{n=2}^{n-2}\left(\frac{1}{n}+\frac{1}{n+1}\right) P_{n}{ }^{1} Q_{n}{ }^{1}-\frac{Q_{0}{ }^{1} \mu \sin \eta}{\lambda^{2}-\mu^{2}}\right\} d \mu .
$$

For $m>n$, by virtue of.

this is reduced to

$$
\int_{-1}^{1} P_{m}{ }^{1} P_{n}{ }^{1} d \mu=0 \quad(m \neq n)
$$

$$
=-\sum \frac{2 m+1}{2 m(m+1)} P_{m}{ }^{1} \int_{-1}^{1} \frac{Q_{0}{ }^{1} \mu \sin \eta \cdot P_{m}{ }^{2}}{\lambda^{2}-\mu^{2}} d \mu
$$

where the integration involved can be evaluated as follows. By means of relations

we have

$$
Q_{0}=\frac{1}{2} \log \frac{\lambda+1}{\lambda-1}, \quad Q_{0}{ }^{1}=Q_{0}{ }^{\prime} \sinh \xi=-\frac{1}{\sinh \xi}, \quad P_{m}{ }^{\prime}=P_{m}{ }^{1} \sin \eta,
$$

$$
\begin{aligned}
\int_{-1}^{1} \frac{Q_{0}{ }^{1} \mu \sin \eta \cdot P_{m}{ }^{1}}{\lambda^{2}-\mu^{2}} d \mu & =-\frac{1}{\sinh \xi} \int_{-1}^{1} \frac{P_{m}{ }^{1} \sin ^{2} \eta \cdot \mu}{\lambda^{2}-\mu^{2}} d \mu \\
& \left.=-\left(\lambda^{2}-1\right)^{1 / 2} \int_{-1}^{1} \frac{P_{m}}{(\lambda-\mu)^{2}} d \mu=2 Q_{m}{ }^{1}, \quad \text { ( } m: \text { even }\right),
\end{aligned}
$$

because of the form found on HoBson p. 116,

Thus, (68) becomes

$$
Q_{n}{ }^{n}(\lambda)=(-1)^{m} \frac{1}{2} m !\left(\lambda^{2}-1\right)^{1 / 2 m} \int_{-1}^{1} \frac{P_{n}(\mu)}{(\lambda-\mu)^{m+1}} d \mu .
$$

$$
=-\sum_{m>n} \frac{2 m+1}{m(m+1)} P_{m}{ }^{1} Q_{m}{ }^{1}
$$


For $m=n$, by means of the relation

(67) is reduced to

$$
\int_{-1}^{1}\left(P_{m}\right)^{2} d \mu=\frac{2 m(m+1)}{2 m+1}
$$

$$
=\frac{2 m+1}{2 m(m+1)}\left\{\frac{2(m+1)}{2 m+1} Q_{m}{ }^{1}-2 Q_{m}{ }^{2}\right\} P_{m}^{1}=-\frac{1}{m+1} Q_{m}{ }^{1} P_{m}{ }^{1} .
$$

For $m<n$, (67) becomes

$$
=\sum_{m<n} \frac{2 m+1}{2 m(m+1)}\left\{\left(\frac{1}{m}+\frac{1}{m+1}\right) \frac{2 m(m+1)}{2 m+1} Q_{m^{1}}-2 Q_{m^{1}}{ }^{1} P_{m}{ }^{1}=0 .\right.
$$

Thus, (59) is reduced to

$$
(\xi \eta)_{c}=\left\{-\sum_{n}^{1} 2 D_{n} Q_{n}{ }^{1} P_{n}{ }^{i}+2 \sum_{m} T_{m}{ }^{\prime} P_{m}{ }^{1}\right\} c^{2} \mu h^{2},
$$

where

$$
T_{m}^{\prime}=-\frac{2 m+1}{m(m+1)} Q^{1} \sum_{n=0}^{m-2} D_{n}-\frac{1}{m+1} Q_{m}{ }^{1} D_{m}
$$

The boundary condition satisfied by $\widehat{\xi \eta}$ being zero at $\xi=\xi_{0}$, we may equate every coefficient of $P_{n}{ }^{1}$ in the form obtained by summing up (57) and (69), to zero, thus,

$$
\left\{\begin{array}{l}
e_{0}-2 D_{0} Q_{0}{ }^{1}+2 T_{0}{ }^{\prime}+e_{2-2}=0, \quad n=0 \\
e_{(n-2)+2}+e_{n}+e_{(n+2)-2}-2 D_{n} Q_{n}{ }^{1}+2 T_{n}{ }^{\prime}=0, \quad n \geqq 2 .
\end{array}\right.
$$

This is another set of infinite simultaneous linear equations relating $B_{n}$ and $D_{n}$.

\section{Numerical calculation (1)}

In order to make out more clearly the nature of stress in the medium, numeri. cal calculations were performed for the case in which the longer axis of the spheroid is three times its shorter one.

In the course of calculation, a table prepared by RIKITAKe was used by his courtesy for the numerical values of $P_{n}, P_{n}{ }^{1}$ and $P_{n}{ }^{\prime \prime}$. But, for those of $Q_{n}, Q_{n}{ }^{1}$ and $Q_{n}{ }^{\prime \prime}$ calculations were worked out, based either on the well-known recurrence: formula, or on the formula in asymptotic form found in HoBson p. 114. that is,

$$
\begin{aligned}
& Q_{n}(\cosh \xi)=\frac{2^{2 n+1}(n !)^{2}}{(2 n+1) !} z^{-(n+1)} F\left(\frac{1}{2}, n+1 ; n+\frac{3}{2}, \frac{1}{z^{2}}\right) \\
& Q_{n}{ }^{1}(\cosh \xi)=\frac{2^{2 n} n !(n+1) !}{(2 n+1) !} z^{-n} \frac{1}{\sinh \xi} F\left(-\frac{1}{2}, n: n+\frac{3}{2}, \frac{1}{z^{2}}\right), \\
& Q_{n}{ }^{\prime \prime}=n(n+1) Q_{n}-\operatorname{coth} \xi Q_{n}{ }^{1}
\end{aligned}
$$

where

$$
z=\cosh \xi+\sinh \xi
$$

The $Q_{n}, Q_{n}{ }^{1}$ and $Q_{n}{ }^{\prime \prime}$ thus obtained are shown in Table 1 . Concerning other cons. tants, numerical values were based on the assumption $\lambda=\mu$ and $A / 2 \mu=1$.

Thus, putting these values into both (46) and (71), we have two sets of infinite number of linear equations with respect to $B_{n}$ and $D_{n}$. Let us refer to the: former set as the first one, and to the latter as the second. Then, from the first: 

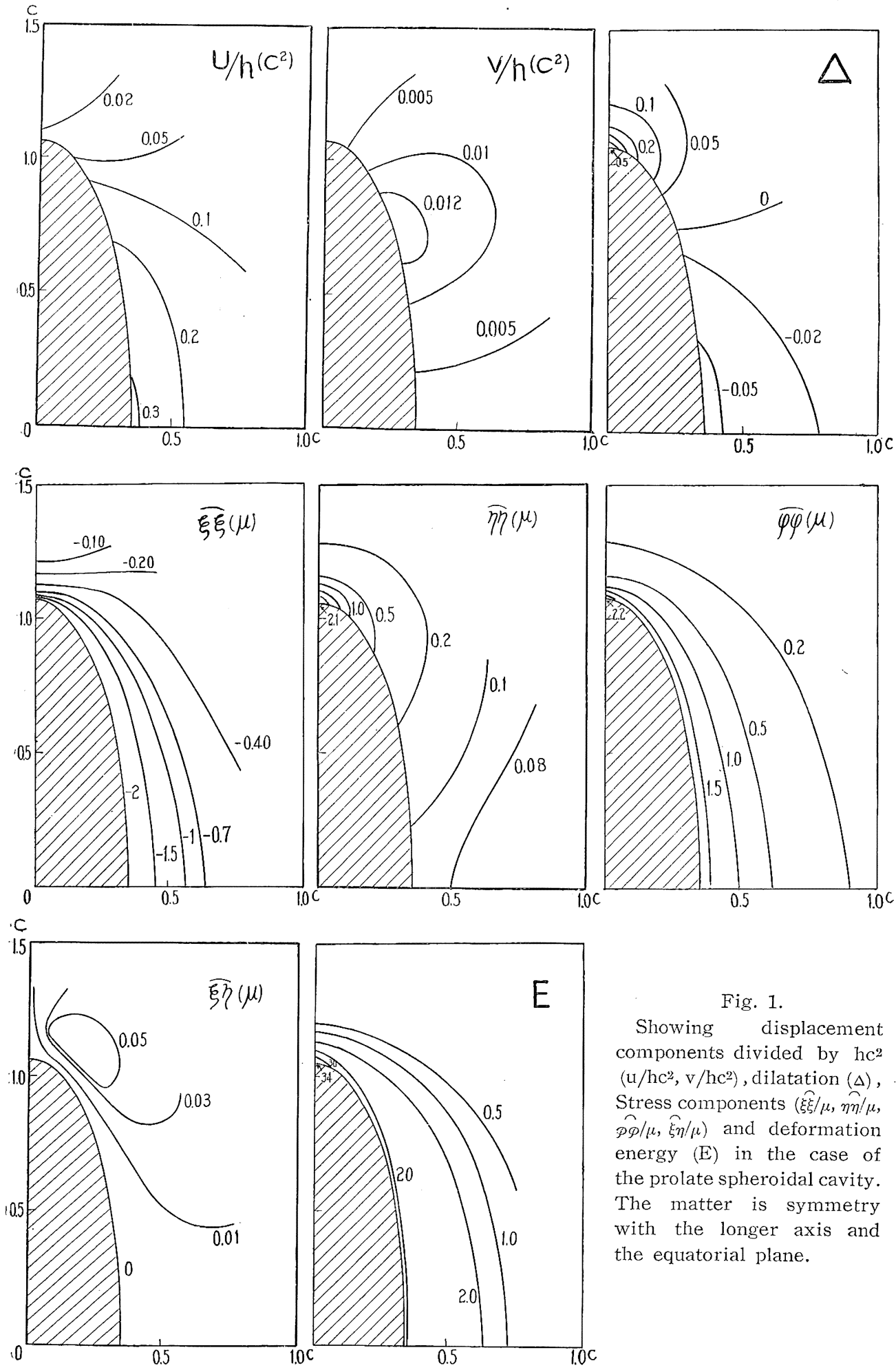

Fig. 1.

Showing displacement components divided by $\mathrm{hc}^{2}$ (u/hc' ${ }^{2}, \mathrm{v} / \mathrm{hc}^{2}$ ), dilatation $(\Delta)$, Stress components $(\widehat{\xi \xi} / \mu, \hat{\eta} / \mu$, $\hat{\rho \varphi} / \mu, \hat{\xi \eta} / \mu)$ and deformation energy (E) in the case of the prolate spheroidal cavity. The matter is symmetry with the longer axis and the equatorial plane. 
set, $D_{n}$ can be easily found to be expressed by $B_{n-2}, B_{n}, B_{n+2}$, and by putting it in $D_{n}$ in the second set, $B_{n}$ can be expressed by $B_{2}$. Again, putting this $B_{n}$ in the first $D_{n}$, we get $D_{n}$ expressed only by $B_{2}$. This rearrangement of the equations was started from the lowest order of $n$ to higher ones successively, but it was not necessary for us to go further than $B_{14}$ or $D_{14}$ to have the value of $B_{2}$ : fixed. This results from a simple assumption that $B_{n} Q_{n}{ }^{\prime \prime}\left(\cosh \xi_{0}\right)$ and $D_{n} Q_{n}{ }^{\prime \prime}\left(\cosh \xi_{0}\right)$ are, as a matter of fact, to converge to zero. The value of $B_{2}$ thus determined has five significant figures. Then, the values of other $B_{n}$ and $D_{n}$ up to $n=14$ were easily obtained by this $B_{2}$ (Table 3 ).

Using these values, we calculated $\Delta, U / c^{2}, V / c^{2}, \widehat{\xi \xi} / \mu, \widehat{\eta \eta} / \mu, \widehat{\varphi \varphi} / \mu$, and $\hat{\xi \eta} / \mu$, the results being tabulated in Table 4, and illustrated in Fig. 1. These values being symmetry concerning the z-axis and the equatorial plane, the spaces shown in the figures are confined to only one quadrant.

According to von Mieses' theory, the breaking of rocks begin when the energy of deformation in the rocks reaches a certain critical value. In our case, the condition of breaking was expressed as follows,

$$
\mu^{2} E=(\widehat{\xi \xi}-\widehat{\eta \eta})^{2}+(\widehat{\eta \eta}-\widehat{\rho \varphi})^{2}+(\widehat{\varphi \varphi}-\widehat{\xi \xi})^{2}+6 \widehat{\xi \eta^{2}} \leqq \frac{3 K^{2} m}{m+1},
$$

where $m$ is Poisson's number and $K$ is a definite constant dependent upon the kind of substance.

If the form of the left-hand side of the above inequality happens to have a maximum value at some point, we may consider this the very point where the first breaking could occur according to the pressure of magma. The numerical values of this form, $E$, at several points in the medium are shown in Table 4 and illustrated in Fig. 1. From this figure, we can deduce that the first point to break is either of the poles of the spheroid.

According to Mohr's theory, the direction in which the breaking would proceed is that of the maximum shear stress, and in this case, it would make an angle of $45^{\circ}$ with the axis of revolution at $\xi=\xi_{0}, \eta=0$, because of hydrostatic pressure acting poun the cavity surface.

\section{Part II. Cavity of Oblate Spheroid}

\section{Particular and complementary solutions}

The procedure of mathematical calculation in this case follows a similar course to the foregoing. Let us employ again the same symbols used in Part I.

Now the relation between spheroidal coordinates $(\xi, \eta, \varphi)$ and orthogonal ones $(x, y, z)$ is given by

$$
z+i \sqrt{x^{2}+y^{2}}=c \sinh (\xi+i \eta)
$$

or

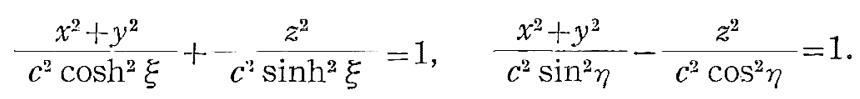

Consequently, $h_{1}, h_{2}$ and $h_{3}$ become 


$$
\left\{\begin{array}{c}
1 \\
h_{1}^{2}=\left(\frac{\partial x}{\partial \xi}\right)^{2}+\left(\frac{\partial y}{\partial \xi}\right)^{2}+\left(\frac{\partial z}{\partial \xi}\right)^{2}=c^{2}\left(\cosh ^{2} \xi-\sin ^{2} \eta\right)=\frac{1}{h_{2}{ }^{2}} \equiv \frac{1}{h^{2}} \\
\frac{1}{h_{3}{ }^{2}}=\left(\frac{\partial x}{\partial p}\right)^{2}+\left(\frac{\partial y}{\partial \varphi}\right)^{2}+\left(\frac{\partial z}{\partial \varphi}\right)^{2}=c^{2} \cosh ^{2} \xi \sin ^{2} \eta .
\end{array}\right.
$$

Further, the equations satisfied by $\Delta$ and $\pi$ become

$$
\left\{\begin{array}{l}
\frac{\partial^{2} \Delta}{\partial \xi^{2}}+\tanh \xi \frac{\partial \Delta}{\partial \xi}+\frac{\partial^{2} \Delta}{\partial \eta^{2}}+\cot \eta \frac{\partial \Delta}{\partial \eta}=0 \\
\frac{\partial^{2} \pi}{\partial \xi^{2}}+\tanh \xi \frac{\partial \pi}{\partial \xi}+\frac{\pi}{\cosh ^{2} \xi}+\frac{\partial^{2} \pi}{\partial \eta^{2}}+\cot \eta \frac{\partial \pi}{\partial \eta}-\frac{\pi}{\sin ^{2} \eta}=0
\end{array}\right.
$$

of which solutions are

$$
\left\{\begin{array}{l}
\Delta_{n}=B_{n} P_{n}(\cos \eta) Q_{n}(i \sinh \xi) \\
\pi_{n}=B_{n b}{ }^{\prime} P_{n}{ }^{1}(\cos \eta) Q_{n}{ }^{1}(i \sinh \xi),
\end{array}\right.
$$

where $B_{n}$ is related to $B_{n}{ }^{\prime}$ by

$$
\begin{aligned}
& (\lambda+2 \mu) B_{n}-2 \mu n(\mathrm{n}+1) B_{n}{ }^{\prime}=0, \quad n \geqq 1 \\
& B_{0}=B_{0}{ }^{\prime}=0 .
\end{aligned}
$$

If we assume $U$ and $V$ as having the same meaning as in (15), we have from (3) and (4),

$$
\left\{\begin{array}{l}
\frac{\Delta}{h^{2}}=\frac{\partial U}{\partial \xi}+\tanh \xi \cdot U+\frac{\partial V}{\partial \eta}+\cot \eta \cdot V \\
2 \pi \\
h^{2}=\frac{\partial V}{\partial \xi}-\frac{\partial U}{\partial \eta}
\end{array}\right.
$$

Cancelling $V$ and $U$ in turn from these, we have

$$
\begin{aligned}
& \partial^{2} U+\frac{\partial}{\partial \xi^{2}}+(\tanh \xi \cdot U)+\cot \eta \frac{\partial U}{\partial \eta}+\frac{\partial^{2} U}{\partial \eta^{2}}=-\frac{\partial}{\partial \xi}\left(\frac{\Delta}{h^{2}}\right)-\frac{\partial}{\partial \eta}\left(\frac{2 \pi}{h^{2}}\right)-\cot \eta \cdot{ }^{2 \pi} \\
& =h_{n}^{2} \\
& \left.\quad \times\left(\cosh ^{2} \xi-\sin ^{2} \eta\right) P_{n}-2 P_{n}{ }^{1} \cos \eta \sin \eta\right\} c^{2} \\
& \frac{\partial^{2} V}{\partial \xi^{2}}+\tanh \xi \frac{\partial V}{\partial \xi}+\frac{\partial^{2} V}{\partial \eta^{2}}+\cot \eta \frac{\partial V}{\partial \eta}-\frac{1}{\sin ^{2} \eta} V=\frac{\partial}{\partial \eta}\left(\frac{\Delta}{h^{2}}\right)+\frac{\partial}{\partial \xi}\left(\frac{2 \pi}{h^{2}}\right)+\tanh \xi \\
& \quad \times \frac{2 \pi}{h^{2}}=B_{n} Q_{n}\left\{-P_{n}{ }^{1}\left(\cosh ^{2} \xi-\sin ^{2} \eta\right)-2 P_{n} \sin \eta \cos \eta\right\} c^{2}+2 B_{n}{ }^{\prime} P_{n}{ }^{1} \\
& \quad \times\left\{n(n+1)\left(\cosh ^{2} \xi-\sin ^{2} \eta\right) Q_{n}+2 Q_{n}{ }^{1} \cosh \xi \sinh \xi\right\} c^{2} .
\end{aligned}
$$

The particular solutions of (78) are

(79) $\left\{\begin{array}{l}U / c^{2}=c_{1} P_{n} Q_{n}{ }^{1} \cosh ^{2} \xi+c_{2} P_{n} Q_{n} \sinh \xi \cosh \xi-c_{1} P_{n} Q_{n}{ }^{1} \sin ^{2} \eta-c_{4} P_{n}{ }^{1} Q_{n}{ }^{1} \cos \eta \sin \eta \\ V / c^{2}=c_{1} P_{n}{ }^{1} Q_{n} \cosh ^{2} \xi+c_{2} P_{n} Q_{n} \cos \eta \sin \eta-c_{1} P_{n}{ }^{1} Q_{n} \sin ^{2} \eta+c_{4} P_{n}{ }^{1} Q_{n}{ }^{1} \sinh \xi \cosh \xi\end{array}\right.$ where the values of $c_{1}, c_{2}$ and $c_{4}$ are the same as given in (20).

The complementary solutions of (78) are

$$
\begin{cases}U_{0} / c^{2}=i D_{0} / \cosh \xi, & U_{n} / c^{2}=D_{n} P_{n} Q_{n}{ }^{1} \\ V_{0} / c^{2}=0, & V_{n} / c^{2}=\cdots D_{n} P_{n}{ }^{1} Q_{n}\end{cases}
$$


The general solution consists of these two kinds of solutions summed up over even values of $n$.

\section{The boundary condition (3).}

The boundary condition is also given by (7). At first, let us consider $(\widehat{\xi \xi})_{\xi=\xi_{0}}$ $=-A$, or (22). Introducing the form of $h$ given by (74) into (22), we have

$$
\left\{\begin{array}{l}
-\frac{A c^{2}}{2 \mu}\left(\cosh ^{2} \xi-\sin ^{2} \eta\right)^{2}=\frac{\lambda c^{2}}{2 \mu}\left(\cosh ^{2} \xi-\sin ^{2} \eta\right)^{2} \Delta \\
+\left\{\begin{array}{l}
\partial U \\
\partial \xi
\end{array}\left(\cosh ^{2} \xi-\sin ^{2} \eta\right)-\sinh \xi \cosh \xi \cdot U-\cos \eta \sin \eta \cdot V\right\}
\end{array}\right.
$$

Substituting the particular solutions given by (79) for $U$ and $V$ involved in (81), the form of the right-hand side becomes

$$
\begin{gathered}
\sum_{n} c^{2}\left(\cosh ^{2} \xi-\sin ^{2} \eta\right)\left[\left\{\left(c_{5}+c_{2}\right) \sinh ^{2} \xi \cdot Q_{n}+\left(c_{1}+c_{2}\right) \sinh \xi \cosh \xi \cdot Q_{n}{ }^{1}+c_{1} \sinh ^{2} \xi \cdot Q_{n}{ }^{\prime \prime}\right\} P_{n}\right. \\
\left.+\left\{\left(c_{5}+c_{2}+n c_{1}\right) Q_{n}+\left(c_{1}+n c_{4}\right) Q_{n}{ }^{\prime \prime}\right\} P_{n} \cos ^{2} \eta-\left(n c_{4} Q_{n}{ }^{\prime \prime}+n c_{1} Q_{n}\right) P_{n-1} \cos \eta\right] .
\end{gathered}
$$

This becomes, by (27) and (29),

$$
=c^{2}\left(\cosh ^{2} \xi-\sin ^{2} \eta\right) \sum\left(d_{n-2}{ }^{\prime} P_{n-2}+d_{n}{ }^{\prime} P_{n}+d_{n+2}{ }^{\prime} P_{n+2}\right),
$$

where

$$
\left\{\begin{aligned}
d_{n-2}{ }^{\prime}= & \alpha_{n-2}^{\prime}\left\{\left(c_{5}+c_{2}+n c_{1}\right) Q_{n}+\left(c_{1}+n c_{4}\right) Q_{n}{ }^{\prime \prime}\right\}-\beta_{n-2}\left(n c_{4} Q_{n}{ }^{\prime \prime}+n c_{1} Q_{n}\right) \\
d_{n}{ }^{\prime}= & \alpha_{n}\left\{\left(c_{5}+c_{2}+n c_{1}\right) Q_{n}+\left(c_{1}+n c_{4}\right) Q_{n}{ }^{\prime \prime}\right\}-\beta_{n}\left(n c_{4} Q_{n}{ }^{\prime \prime}+n c_{1} Q_{n}\right) \\
& +\left(c_{1}+c_{2}\right) \sinh \xi \cosh \xi \cdot Q_{n}{ }^{1}+c_{1} \sinh ^{2} \xi \cdot Q_{n}{ }^{\prime \prime} \\
d_{n+2}{ }^{\prime}= & \alpha_{n+2}\left\{\left(c_{5}+c_{2}+n c_{1}\right) Q_{n}+\left(c_{1}+n c_{4}\right) Q_{n}{ }^{\prime \prime}\right\}
\end{aligned}\right.
$$

further, $\alpha_{n}$ and $\beta_{n}$ are given by (30), and $c_{5}$, by (26).

On the other hand, if we substitute the complementary solutions given by (79) for $U$ and $V$ in (81), the form of the right-hand side becomes

$$
\left\{\begin{array}{c}
\sum_{n} c^{2}\left(\cosh ^{2} \xi-\sin ^{2} \eta\right)\left\{D_{n} P_{n} Q_{n}{ }^{\prime \prime}-P_{n}\left(\sinh \xi \cosh \xi \cdot Q_{n}{ }^{1} P_{n}-\cos \eta \sin \eta \cdot Q_{n} P_{n}{ }^{1}\right)\right. \\
\left./\left(\cosh ^{2} \xi-\sin ^{2} \eta\right)\right\} .
\end{array}\right.
$$

Now, let us expand (84) in $P_{m}$ series. In a similar manner to Part I, we have from (34),

$$
\left\{\begin{array}{c}
\sinh \xi \cosh \xi \cdot Q_{n}{ }^{1} P_{n}-\cos \eta \sin \eta \cdot Q_{n} P_{n}{ }^{1}=n\left(-\lambda^{2}+\mu^{2}\right) P_{n} Q_{n}+n\left(\lambda P_{n} Q_{n-1}-\mu P_{n-1} Q_{n}\right) \\
=-(n+1)\left(-\lambda^{2}+\mu^{2}\right) P_{n} Q_{n}+(n+1)\left(-\lambda P_{n} Q_{n+1}+\mu P_{n+1} Q_{n}\right),
\end{array}\right.
$$

where $\lambda=i \sinh \xi$.

Further, from (37) and taking into consideration $-\lambda^{2}+\mu^{2}=\cosh ^{2} \xi-\sin ^{2} \eta$, we may transform the right hand-side of the above equation into

$$
\left\{\begin{array}{l}
=n\left(\cosh ^{2} \xi-\sin ^{2} \eta\right) P_{n} Q_{n}+\frac{n(n-1)}{2 n-1}\left(P_{n 2} Q_{n-2}-P_{n-2} Q_{n}\right) \\
=-(n+1)\left(\cosh ^{2} \xi-\sin ^{2} \eta\right) P_{n} Q_{n}+\frac{(n+1)(n+2)}{2 n+3}\left(P_{n+2} Q_{n}-P_{n} Q_{n+2}\right) .
\end{array}\right.
$$

From these two forms we have a recurrence formula

$$
\left\{\begin{array}{l}
\frac{(n+1)(n+2)}{2 n+3}\left(P_{n+2} Q_{n}-P_{n} Q_{n+2}\right) \\
\quad=(2 n+1)\left(\cosh ^{2} \xi-\sin ^{2} \eta\right) P_{n} Q_{n}+\frac{n(n-1)}{2 n-1}\left(P_{n} Q_{n-2}-P_{n-2} Q_{n}\right) .
\end{array}\right.
$$


Using this relation repeatedly, we have

$$
=\sum_{n=2}^{n}(2 n+1) P_{n} Q_{n}\left(\cosh ^{2} \xi-\sin ^{2} \eta\right)+\frac{2}{3}\left(P_{2} Q_{0}-P_{0} Q_{2}\right) .
$$

By means of (41), the last term in the right-hand side may be written as: $\left(-\lambda^{\prime \prime}+\mu^{2}\right) Q_{0}+\lambda$. Hence, we have

$$
\left\{\begin{array}{l}
\frac{\cosh \xi \sinh \xi \cdot Q_{n}{ }^{1} P_{n}-\cos \eta \sin \eta \cdot Q_{n} P_{n}{ }^{1}}{\cosh ^{2} \xi-\sin ^{2} \eta} \\
=\sum_{m} \frac{2 m+1}{2} P_{m} \int_{-1}^{1} P_{m} \frac{\sinh \xi \cosh \xi \cdot Q_{n}{ }^{1} P_{n}-\cos \eta \sin \eta \cdot Q_{n} P_{n}{ }^{1}}{\cosh ^{2} \xi-\sin ^{2} \eta} d \mu \\
=\sum_{m} \frac{2 m+1}{2} P_{m} \int_{-1}^{1} P_{m}\left\{n P_{n} Q_{n}+\sum_{n=2,4,6}^{n}(2 n-3) P_{n-2} Q_{n-2}+\frac{\lambda}{\cosh ^{2} \xi-\sin ^{2} \eta}\right\} d \mu
\end{array}\right.
$$

Calculating in a similar manner to that in (42a), (42b), (42c), we may reduce the right-hand side of (89) to

$$
\text { (90) }= \begin{cases}-\sum_{m=n+2}^{\infty}(2 m+1) Q_{m} P_{m}, & m>n \\ -(m+1) Q_{m} P_{m} & m=m \\ 0 & m<n,\end{cases}
$$

where $m$ is an even number, and for the odd number the corresponding values are all zero.

Therefore, (84) may be expanded by $P_{n}$ as follows,

$$
c^{2}\left(\cosh ^{2} \xi-\sin ^{2} \eta\right)\left(\sum_{n} D_{n} P_{n} Q_{n}^{\prime \prime}-\sum_{m} Y_{m} P_{m}\right),
$$

where

$$
Y_{m}=D_{n}\left\{-\sum_{n=0}^{m-2}(2 m+1) Q_{n}-(m+1) Q_{m}\right\} .
$$

Equating (91) plus (82) to the left-hand side of (81) and putting the coefficient of the same $P_{n}$ of both sides of the equation to be equal, we have

$$
\left\{\begin{array}{l}
d_{2-2}+\left(D_{0} Q_{0}{ }^{\prime \prime}-Y_{0}\right)=-\frac{A}{2 \mu}\left(\sinh ^{2} \xi+\frac{1}{3}\right) \\
d_{2}+d_{4-2}+\left(D_{2} Q_{2}{ }^{\prime \prime}-Y_{2}\right)=-\frac{2}{3} \frac{A}{2 \mu} \\
d_{n+2}+d_{(n+2)}+d_{(n+4)-2}+\left(D_{n+2} Q_{n+2}{ }^{\prime \prime}-Y_{n+2}\right)=0, \quad n \geqq 2 .
\end{array}\right.
$$

Next, let us consider about $\widehat{\xi \eta}=0$ at $\xi=\xi_{0}$. From (47) and (74) we have

$$
\widehat{\xi \eta}=\mu h^{2}\left\{\left(\frac{\partial V}{\partial \xi}+\frac{\partial U}{\partial \eta}\right)-2 c^{2} h^{2}(V \cosh \xi \sinh \xi-U \cos \eta \sin \eta)\right\} .
$$

If only the particular solutions of $U$ and $V$ given by (79) are put in this, we: get,

$$
\begin{aligned}
(\widehat{\xi \eta})_{p}= & \sum_{n} \mu h^{2} c^{3}\left\{-c_{4} P_{n}{ }^{1} Q_{n}{ }^{1}\left(\sinh ^{2} \xi-\cos ^{2} \eta\right)+\left(n(n+1) c_{4}-c_{2}\right)\right. \\
& \left.\times\left(-Q_{n}{ }^{1} P_{n} \cos \eta \sin \eta+P_{n}{ }^{1} Q_{n} \sinh \xi \cosh \xi\right)\right\}
\end{aligned}
$$

Referring to (52) and (55), we may transform this into

$$
=\sum_{n} c^{2} \mu h^{2}\left(e_{n+2}{ }^{\prime} P_{n+2}{ }^{1}+e_{n}{ }^{\prime} P_{n}{ }^{1}+e_{n-2}{ }^{\prime} P_{n-2}{ }^{\mathrm{i}}\right) \text {, }
$$

where 


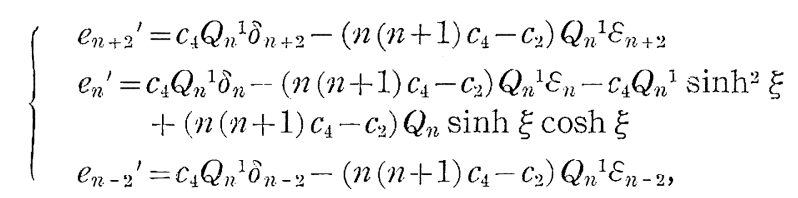

in which $\delta_{n}$ and $\varepsilon_{n}$ are given by (53) and (56).

On the other hand, if only the complmentary solutions given by (80) are substituted for $U$ and $V$, the right-hand side of (94) takes the form

$$
\left\{\begin{aligned}
(\xi \eta)_{c}= & \sum \mu h h^{2} c^{2}\left\{-2 D_{n} Q_{n}{ }^{1} P_{n}{ }^{1}+2\left(D_{n} P_{n}{ }^{1} Q_{n} \cosh \xi \sinh \xi+D_{n} P_{n} Q_{n}{ }^{1} \cos \eta\right.\right. \\
& \left.\times \sin \eta) /\left(\cosh ^{2} \xi-\sin ^{2} \eta\right)\right\} .
\end{aligned}\right.
$$
Part I.

In expanding the second term by $P_{m}$, we may follow the same course as in

From (61), we can get

$$
\left\{\begin{array}{l}
\cosh \xi \sinh \xi \cdot P_{n}{ }^{1} Q_{n}+\cos \eta \sin \eta \cdot P_{n} Q_{n}{ }^{1} \\
\quad=\frac{1}{n}\left(-\lambda^{2}+\mu^{2}\right) P_{n}{ }^{1} Q_{n}{ }^{1}+\frac{1}{n}\left(\lambda P_{n}{ }^{1} Q_{n+1}{ }^{1}-\mu P_{n-1}{ }^{1} Q_{n}{ }^{1}\right) \\
\quad=\frac{-1}{n+1}\left(-\lambda^{2}+\mu^{2}\right) P_{n}{ }^{1} Q_{n}{ }^{1}+\frac{1}{n+1}\left(\mu P_{n+1}{ }^{1} Q_{n}{ }^{1}-\lambda P_{n}{ }^{1} Q_{n+1}{ }^{1}\right)
\end{array}\right.
$$

By (64), we may transform both the above right-hand side forms into

$$
\left\{\begin{array}{l}
={ }^{1}\left(\cosh ^{2} \xi-\sin ^{2} \eta\right) P_{n}{ }^{1} Q_{n}{ }^{1}+\frac{1}{2 n-1}\left(P_{n}{ }^{1} Q_{n-2}{ }^{1}-P_{n-2}{ }^{1} Q_{n}{ }^{1}\right) \\
=-\frac{1}{n+1}\left(\cosh ^{2} \xi-\sin ^{2} \eta\right) P_{n}{ }^{1} Q_{n}{ }^{1}+\frac{1}{2 n+3}\left(P_{n+2}{ }^{1} Q_{n}{ }^{1}-P_{n}{ }^{1} Q_{n+2}{ }^{1}\right) .
\end{array}\right.
$$

From these forms, we have a recurrence formula

$$
\left\{\begin{aligned}
\frac{1}{2 n+3}\left(P_{n}{ }^{1} Q_{n+2}{ }^{1}-P_{n+2}{ }^{1} Q_{n}{ }^{1}\right)= & -\left(\begin{array}{c}
1 \\
n
\end{array}+\frac{1}{n+1}\right)\left(\lambda^{2}-\mu^{2}\right) P_{n}{ }^{1} Q_{n}{ }^{1} \\
& +\frac{1}{2 n-1}\left(P_{n-2}{ }^{1} Q_{n}{ }^{1}-P_{n}{ }^{1} Q_{n-2}{ }^{1}\right)
\end{aligned}\right.
$$

By repeated use of this relation, its right hand side becomes

$$
=-\sum_{n=2}^{n}\left(\frac{1}{n}+\frac{1}{n+1}\right) P_{n}{ }^{1} Q_{n}{ }^{1}\left(\lambda^{2}-\mu^{2}\right)+\frac{1}{3}\left(P_{0}{ }^{1} Q_{2}{ }^{1}-P_{2}{ }^{1} Q_{0}{ }^{1}\right),
$$

where the last term is equal to $-\sin \eta \cos \eta \cdot Q_{0}{ }^{1}$.

Therefore, we have

$$
\left\{\begin{aligned}
& \frac{P_{n}{ }^{1} Q_{n} \cosh \xi \sinh \xi+P_{n} Q_{n}{ }^{1} \cos \eta \sin \eta}{\cosh ^{2} \xi-\sin ^{2} \eta} \\
&=\sum_{m} \frac{2 m+1}{2 m(m+1)} P_{m} \int_{-1}^{1} P_{m}{ }^{1} \frac{P_{n}{ }^{1} Q_{n} \cosh \xi \sinh \xi+P_{n} Q_{n}{ }^{1} \cos \eta \sin \eta}{\cosh ^{2} \xi-\sin ^{2} \eta} d \mu . \\
&=\sum_{m} \frac{2 m+1}{2 m(m+1)} P_{m}{ }^{1} \int_{-1}^{1} P_{m}{ }^{1} \\
& \times\left\{\frac{1}{n} P_{n}{ }^{1} Q_{n}{ }^{1}+\sum_{n=2}^{n-2}\left(\frac{1}{n}+\frac{1}{n+1}\right) P_{n}{ }^{1} Q_{n}{ }^{1}+\frac{\sin \eta \cos \eta \cdot Q_{0}{ }^{1}}{\cosh ^{2} \xi-\sin ^{2} \eta}\right\} d \mu .
\end{aligned}\right.
$$

Following the same procedure as used in evaluating (67), we get 


$$
\text { (103) }= \begin{cases}-\sum_{m=n+2}^{\infty} \frac{2 m+1}{m(m+1)} P_{m}{ }^{1} Q_{m}{ }^{1}, & m n, \\ \frac{1}{m+1} P_{m}^{1} Q_{m}{ }^{1}, & m=n, \\ 0, & m<n .\end{cases}
$$

Then (97) is reduced to

$$
(\widehat{\xi \eta})_{c}=c^{2} \mu h^{2}\left(-\sum_{n} 2 D_{n} Q_{n}{ }^{1} P_{n}{ }^{1}+2 \sum_{m} Y_{m}{ }^{\prime} P_{m}{ }^{1}\right),
$$

where

$$
Y_{m}{ }^{\prime}=-\frac{2 m+1}{m(m+1)} Q_{m:}{ }^{m} \sum_{n=0}^{m-2} D_{n} \cdot-\frac{1}{m+1} Q_{m}{ }^{1} D_{m}
$$

Summing up both forms of (95) and (104) and putting this, or each coefficient of $P_{n}{ }^{1}$. equal to zero, we have

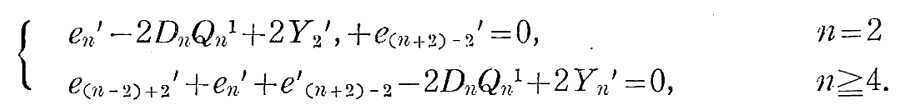

\section{Numerical calculation (2)}

Numerical calculations were made in the same manner as in Part I. The assumption is also made that the ratio of the longer axis to the shorter one is $3: 1, \lambda=\mu$, and $A / 2 \mu=1$.

Numerical values of $Q_{n}(i \sinh \xi), Q^{1}(i \sinh \xi)$ and $Q_{n}^{\prime \prime}$ are derived from the recurrence formula or the asymptotic forms found in Hobson p. 286, that is,

$$
\begin{aligned}
Q_{n}(\mu) & =\frac{2^{2 n+1}(n !)^{2}}{(2 n+1) !} z^{-n} \frac{1}{\sqrt{z^{2}-1}} F\left(\frac{1}{2}, \frac{1}{2} ; n+\frac{3}{2}, \frac{1}{1-z^{2}}\right), \\
Q_{n}{ }^{1}(\mu) & =-\frac{2^{2 n+2} n !(n+1) !}{(2 n+1) !} \frac{i \cosh \xi}{z^{n-1}\left(z^{2}-1\right)^{2 / 3}} F\left(\frac{3}{2},-\frac{1}{2} ; n+\frac{3}{2}, \frac{1}{1-z^{2}}\right), \\
Q_{n}{ }^{\prime \prime} & =n(n+1) Q_{n}-\tanh \xi \cdot Q_{n}{ }^{1},
\end{aligned}
$$

where

$$
\mu=i \sinh \xi \text { and } z=\mu+\sqrt{\mu^{2}-1}
$$

The $Q_{n}, Q_{n}{ }^{1}$ and $Q_{n}{ }^{\prime \prime}$ thus obtained are shown in Table 2.

The values of $B_{n}, D_{n}, U / c^{2}, V / c^{2}, \Delta, \widehat{\xi \xi} / \mu, \widehat{\eta \eta} / \mu, \widehat{\phi \varphi} / \mu$ and $\widehat{\xi \eta} / \mu$ thus calculated are shown in Tables 3 and 5 , and the form in (72), or $E$, in Table 5. These are also illustrated in Fig. 2.

If these figures are compared with the corresponding ones for the prolate cavity by putting the $\sqrt{x^{2}+y^{2}}$ axis for the oblate upon the $z$-axis for the prolate, they may be found to resemble each other closely, though the former values and their variations are generally larger than the latter. Therefore, characteristics shown in these two cases may be explained in common by the following description.

As for the displacement in the medium neighbouring the cavity, $U$ has everywhere an outward sense with larger value at places nearer to the shorter axis of the cavity: $V$ is everywhere of sense toward the shorter axis. having a maximum 

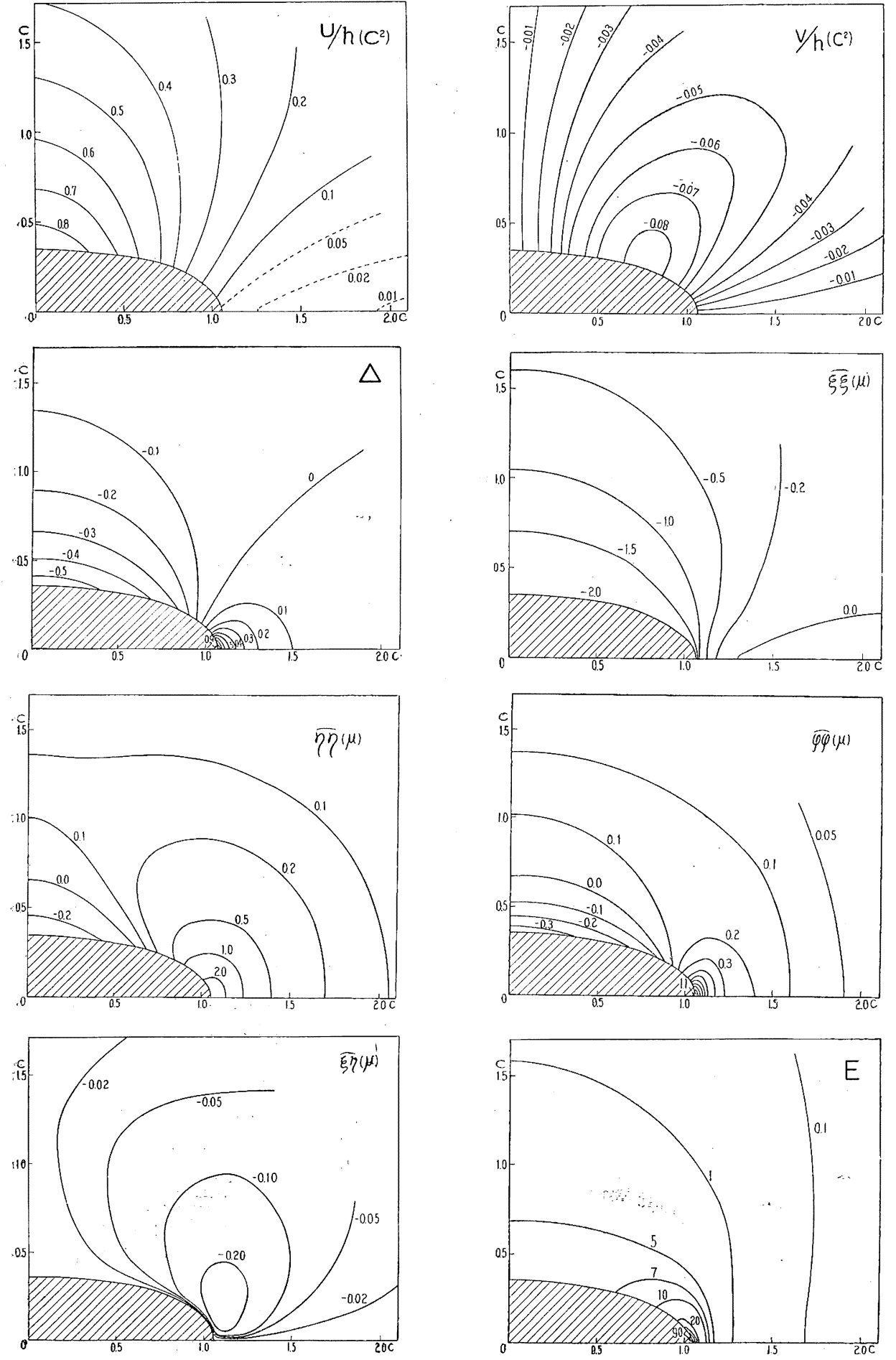

Fig. 2. Showing displacement components divided by $\mathrm{hc}^{2}\left(\mathrm{u} / \mathrm{hc}^{2}, \mathrm{v} / \mathrm{hc}^{\mathrm{2}}\right)$, dilatation $(\Delta)$, stress components $(\hat{\xi} \xi / \mu, \hat{\eta} \eta / \mu, \hat{\rho} \varphi / \mu, \hat{\xi \eta} / \mu)$ and deformation energy (E) in the case of the oblate spheroidal cavity. The matter is symmetry with the shorter axis and the equatorial plane. 
value at the point approximately $\eta=45^{\circ}$ on the cavity. Its value becomes smaller farther from it, attaining to zero on both axes, $z$ and $\sqrt{x^{2}+y^{2}}$.

$\Delta$ has the maximum value at the point on the cavity intersecting with its longer axis, where its sense is of expansion, while it has the minimum value at the point on the cavity intersecting with its shorter axis, where its sense is of contraction. Separating these expansion and contraction regions, there is a neutral line that starts from the point on the cavity wall located at $\eta=45^{\circ}$ for the prolate case and $65^{\circ}$ for the oblate case.

As for stress, $\widehat{\xi \xi}$ is everywhere of compression sense, its value becoming larger at places nearer to the shorter axis of the cavity. $\widehat{\eta \eta}$ and $\widehat{\phi \varphi}$ have the maximum values at the point on the cavity wall intersecting with the longer axis where their senses are of tension, while the minimum values appear at the point on the cavity wall intersecting with the shorter axis, where it remains to be tension for prolate case, but becomes compression for the oblate case. This difference is remarkable. $\widehat{\xi \eta}$ is everywhere of the same sense, that is, shear acts on the plane $\xi=$ const. in the origin side toward the shorter axis, and its maximum value appears in the neighbourhood of the longer axis slightly apart from the cavity wall. The maximum value of $E$ occurs at the point on the cavity wall intersecting with the longer axis. Returning to the oblate case we may find, from Fig. 2, that. points on the equatorial circle of the spheroid is the most liable to break first in response to a sufficiently high pressure of magma. By a similar consideration to case dealt with in Part I, it is easily inferred that the resultant fissure would run. in the direction making the angle of $45^{\circ}$ with the axis of revolution.

\section{Discussion}

We have seen in the above two examples that the breaking of the spheroidal cavity would occur at either of the two poles for the prolate one and at some arc on the equatorial circle for the oblate one. Though these examples are of a special ellipticity, the above-mentioned tendency may be considered to hold for any other ellipticity. In other words, it may be said that the point where the curvature of wall has the minimum radius is the point most liable to break.

Further, it could be imagined that the maximum value of the deformation. energy on the wall of cavity may increase as the ellipticity increases, that is, as the oblate spheroid becomes more flat or the prolate one more slender. And so is it with the possibility of breaking.

It is notable that the value of $E$ diminishes rapidly with $\xi$. Consequently a. fracture, if it occurred, would not proceed to so great a distance from the cavity wall at one time, releasing a part of magma pressure. Then it is very probable that the second and third breaking would happen successively.

It is remarkable that while the displacement in the medium is proportional to $c^{2}$, or to the square of the dimension of cavity, the stress in the medium is independent of $c$. Therefore, the pressure sufficient to cause a breaking does not. depend on $c$ so long as its ellipticity is constant.

Comparison of Figs. 1 and 2 shows that the highest deformation energy appearing in the case of the prolate type cavity, is not so large as those appearing in. the case of the oblate one. From this, it is clear which type of cavity is liable to break if both cavities are under a common hydrostatic pressure. The calculation 
shows that if the oblate type could break with pressure $A$, about $1.7 A$ would be necessary for the prolate one to break. Further, the deformation energy being concentrated along a circle, breaking would occur more easily to the oblate cavity than to the prolate one in which the concentration appears only in the vicinities of the two points.

At the instant of breaking, the mode in which the displacement and stress are distributed would transfer from ours into another one, and in its transitional state, some dynamic force would appear. What force would happen then is another problem to be solved. But roughly speaking, as the movement on either side of the fissure, or fault, would start in opposite directions to each other, it may be imagined in the case of the oblate spheroid that an outburst of the cavity wall adjoining to the fault surface on the pole side would occur, giving a force of shock type to the medium in the direction of approximately zaxis. And at the same time an equal and opposite force would appear in the wall of opposite sides across the cavity space in accordance with the law of momentum conservation. On the other hand, more or less pulling force would be caused in the region adjoining the fault surface on the side of the equatorial plane. The original pulling force would also form a couple with another one equal and opposite to it satisfying the law of momentum conservation. Besides this, there is another way for pulling force to occur. Considering that the couple of forces generated in the direction $\pm z$ acts as if to tear the cavity along its equatorial circle, it would be probable that a force is caused there which is symmetrical concerning the z-axis, and acts in the pull sense. Thus, these forces would be distributed as a whole in a cone type with a push sense inside of it, though what an angle the apex of the cone would make is another problem.

Following a similar codsideration, we may suppose that the cone type distribution of force with pull sense inside of the cone could occur in the case of the prolate type. As stated above, the result of our theory that the breaking of the prolate type is less liable to occur than the former agrees qualitatively with the fact that an earthquake having the second kind of cone-type distribution in initial motion is rare in nature.

Aknowiedgement - The authors heartily appreciate Mr. OGAWARA's valuable suggestion about convergency of the solution.

\section{References}

[1] Matuzawa, T. 1953: Feldthorie der Erdbeben. Bull. Earthq. Res. Inst. 31, p.179.

[2] Ishnoro, M. 1929: Sur le Mecanisme de la Production des Ondes Sismiques. (en japonais). Bull. Earthq. Res. Inst. 6, p. 127.

[3] TAKagr: S. 1943: On the Origin of Earthquake (I), (in Japanese) Quat. Journ. Seis. 13, p. 1.

[4] Wabatr, K. 1937: On the Mechanism of Earthquakes and the Propagation of Seismic Waves. (in Japanese). Journ. Met. Soc. Japan. 2nd Ser. 15, p. 295.

[5] Ishimoto, M. 1932: Existence d'une Source Quadruple au Foyer Sismique d'après l'étude de la Distribution des Mouvements Initiaux des Secousses Sismiques. Bull. Earthq. Res. Inst. 10, p. 449.

[6] SaKaTA, K. 1941: On an Earthquake of "Cone-tye" Mechanism with Pull Sesne Inside (in Japanese). "Umi to Sora” 21, p. 176.

[7] Matuzawa, T. 1942: Seismometrische Untersuchungen des Erdbebens vom 3. Marz 1933. IV. Raumverteilingen der Wellenstrahlung aus dem Herd. Bull. Earthq. Res. Inst. 20, p. 162 . 
Table 1.

\begin{tabular}{|c|c|c|c|c|c|c|c|c|c|}
\hline \multirow[b]{2}{*}{$n$} & \multicolumn{3}{|c|}{$\cosh \xi=x=1 . c 6$} & \multicolumn{3}{|c|}{$x=1.15$} & \multicolumn{3}{|c|}{$x=1.25$} \\
\hline & $Q_{n}(x)$ & $Q_{n^{1}(x)}$ & $Q_{n}^{\prime \prime}(x)$ & $Q_{n}(x)$ & $Q_{n}{ }^{1}(x)$ & $Q_{n}{ }^{\prime \prime}(x)$ & $Q_{n}(x)$ & ${\overline{Q_{n}}}^{1}(x)$ & $Q_{n}^{\prime \prime}(x)$ \\
\hline $\begin{array}{l}0 \\
2 \\
4 \\
6 \\
8\end{array}$ & $\begin{array}{l}1.768050 \\
c .505797 \\
0.197334 \\
0.083937 \\
0.037226\end{array}$ & $\begin{array}{l}-2.84440 \mathrm{i} \\
-1.922443 \\
-1.157281 \\
-0.662928 \\
-0.369235\end{array}$ & $\begin{array}{l}8.576052 \\
8.831073 \\
7.435952 \\
5.524113 \\
3.793516\end{array}$ & $\begin{array}{l}1.331292 \\
0.250307 \\
0.065369 \\
0.018691 \\
0.005582\end{array}$ & $\begin{array}{l}-1.760902 \\
-0.856272 \\
-0.356332 \\
-0.139550 \\
-0.052889\end{array}$ & $\begin{array}{l}3.565892 \\
3.235824 \\
2.028961 \\
1.067616 \\
0.508991\end{array}$ & $\begin{array}{l}1.098611 \\
0.150565 \\
0.028905 \\
0.006087 \\
0.001340\end{array}$ & \begin{tabular}{|l|}
-1.333333 \\
-0.493436 \\
-0.153095 \\
-0.044468 \\
-0.012474
\end{tabular} & $\begin{array}{l}2.222222 . \\
1.725867 \\
0.833254 \\
0.329755 \\
0.117248\end{array}$ \\
\hline $\begin{array}{l}10 \\
12 \\
14 \\
16 \\
18 \\
20\end{array}$ & $\begin{array}{l}0.016921 \\
0.007822 \\
0.003658 \\
0.001726 \\
0.0008196 \\
0.0003915\end{array}$ & $\begin{array}{l}-0.201956 \\
-0.109065 \\
-0.058268 \\
-0.030988 \\
-0.016363 \\
-0.008600\end{array}$ & $\begin{array}{l}2.470232 \\
1.549016 \\
0.943780 \\
0.562770 \\
0.329643 \\
0.190339\end{array}$ & $\begin{array}{l}0.001710 \\
0.0005330 \\
0.0001681 \\
0.0000535 \\
0.0000172\end{array}$ & $\begin{array}{l}-0.019636 \\
-0.007187 \\
-0.0026 C 4 \\
-0.0009358 \\
-0.00(3344\end{array}$ & $\begin{array}{l}0.227873 \\
0.097698 \\
0.040576 \\
0.016450 \\
0.006542\end{array}$ & $\begin{array}{l}0 . \operatorname{coc} 3026 \\
0.00 C C 695 \\
0.0000162 \\
0.0000038\end{array}$ & $\begin{array}{l}-0.003424 \\
-0.0009261 \\
-0.0002478 \\
-0.0000658\end{array}$ & $\begin{array}{l}0.038993 \\
0.012392 \\
0.003811 \\
0.001143\end{array}$ \\
\hline
\end{tabular}

Table 2.

(unit : i)

\begin{tabular}{|c|c|c|c|c|c|c|c|c|c|}
\hline \multirow{2}{*}{$n$} & \multicolumn{3}{|c|}{$\cosh \xi=1.06$} & \multicolumn{3}{|c|}{$\cosh \xi=1.10$} & \multicolumn{3}{|c|}{$\cosh \xi=: .15$} \\
\hline & $Q_{n}(i \sinh \xi)$ & $Q_{n}^{1}(i \sinh \xi)$ & $\mid Q_{n}^{\prime \prime}(i \sinh \xi)$ & $Q_{1}(i \sin n \xi)$ & $Q_{n}{ }^{1}($ isinh & $Q_{n}^{\prime \prime}(i \sinh \xi)$ & $Q_{n}(i \sinh \xi)$ & & $\overline{\mid} Q_{n}^{\prime \prime}(i \sin h \xi)$ \\
\hline $\begin{array}{l}0 \\
2 \\
4 \\
6 \\
8\end{array}$ & $\begin{array}{r}-1.232698 \\
0.317558 \\
-0.120245 \\
0.05042 .1 \\
-0.022172\end{array}$ & $\begin{array}{r}0.943430 \\
-0.858434 \\
0.563758 \\
-0.336852 \\
0.192413\end{array}$ & \begin{tabular}{r|r|} 
& -0.312894 \\
& 2.190064 \\
& -2.591875 \\
& 2.229412 \\
& -1.666170
\end{tabular} & \begin{tabular}{r|r}
4 & -1.141094 \\
0.242607 \\
5 & -0.075487 \\
0.025989 \\
-0.009381
\end{tabular} & $\begin{array}{r}0.909094 \\
-0.665286 \\
0.356977 \\
-0.174724 \\
0.08: 804\end{array}$ & $\begin{array}{r}-0.378727 \\
1.732801 \\
-1.658465 \\
1.164312 \\
-0.709540\end{array}$ & $\mid \begin{array}{r}-1.054320 \\
0.185532 \\
-0.047510 \\
0.013465 \\
-0.004001\end{array}$ & $\mid$\begin{tabular}{r|}
0.869566 \\
-0.514784 \\
0.226412 \\
$-0.091 C 30$ \\
0.035034
\end{tabular} & $\begin{array}{r}-0.429408 \\
1.367401 \\
-1.062009 \\
0.610496 \\
-0.305342\end{array}$ \\
\hline $\begin{array}{l}10 \\
12 \\
14 \\
16 \\
18 \\
20\end{array}$ & $\begin{array}{r}0.010023 \\
-0.004614 \\
0.002151 \\
-0.001013 \\
0.0004801 \\
-0.0002289\end{array}$ & $\begin{array}{r}-0.107011 \\
0.058482 \\
-0.031566 \\
0.016881 \\
-0.008964 \\
9 \\
9.004732\end{array}$ & $\begin{array}{r}1.138075 \\
-0.739209 \\
0.462235 \\
-0.281007 \\
0.167154 \\
-0.097722\end{array}$ & $\begin{array}{r}0.003481 \\
-0.001315 \\
0.0005 C 32 \\
-0.0001944 \\
0.0000756\end{array}$ & \begin{tabular}{|r|}
-0.037309 \\
\\
$2 \mid 0.016724$ \\
4 \\
$4-0.007405$ \\
$6-0.003249$ \\
-0.001415
\end{tabular} & $\begin{array}{r}0.398457 \\
-0.212129 \\
0.108759 \\
-0.054222 \\
0.026452\end{array}$ & $\begin{array}{r}0.001222 \\
-0.0003798 \\
0.0001196 \\
-L \cdot 0000380 \\
0.0000: 22\end{array}$ & \begin{tabular}{|c|}
-0.013139 \\
0.004844 \\
-0.001764 \\
0.0006367 \\
-0.0002282
\end{tabular} & $\begin{array}{r}0.140865 \\
-0.061639 \\
0.025981 \\
-0.01 C 652 \\
2 \quad 0.004274\end{array}$ \\
\hline \multirow[b]{2}{*}{$n$} & \multicolumn{3}{|c|}{$\cosh \xi=1.25$} & \multicolumn{3}{|c|}{$\cosh \xi=1.50$} & \multicolumn{3}{|c|}{$\cosh \xi=2 . c 0$} \\
\hline & $Q_{n}(i$ & $Q$ & $Q_{n}{ }^{\prime \prime}(i \sinh \xi)$ & $Q_{n}(i \sinh \xi)$ & $\bar{Q}_{n}{ }^{1}(i \sin$ & $\mid h \xi)$ & b) & 18 & $h \xi)$ \\
\hline $\begin{array}{l}0 \\
2 \\
4 \\
6 \\
8\end{array}$ & $\begin{array}{r}-0.927295 \\
0.121053 \\
-0.022930 \\
0.004798 \\
-0.001052\end{array}$ & $\begin{array}{r}0.800000 \\
-0.341982 \\
0.110418 \\
-0.03268: \\
0.009268\end{array}$ & $\mid \begin{array}{r}-0.480 c 00 \\
0.931507 \\
-0.524845 \\
0.221131 \\
-0.08 .317\end{array}$ & $\begin{array}{c}-0.729728 \\
0.055823 \\
-0.066209 \\
0.0007590 \\
-0.0000972\end{array}$ & $\begin{array}{c}0.666667 \\
-0.16 ! 962 \\
0.030321 \\
-0.005222 \\
0.0008629\end{array}$ & $\begin{array}{r}-0.4969 C 4 \\
0.455655 \\
-0.146786 \\
0.03577: \\
-0.007641\end{array}$ & $\left|\begin{array}{c}-0.523600 \\
0.019918 \\
-0.001088 \\
0.000 C 655 \\
-0.000 C 041\end{array}\right|$ & $\mid \begin{array}{c}0.500000 \\
-0.058602 \\
0.005371 \\
-0.0004542 \\
0.0000369\end{array}$ & $\begin{array}{r}-0.433013 \\
0.270257 \\
-0.026464 \\
0.003143 \\
-0.0004290\end{array}$ \\
\hline $\begin{array}{l}10 \\
12 \\
14\end{array}$ & $\left|\begin{array}{r}0.0002371 \\
-0.0000544 \\
0.0000126\end{array}\right|$ & $\left|\begin{array}{c}-0.002562 \\
0.0006966 \\
-0.000: 871\end{array}\right|$ & $\begin{array}{r}0.027620 \\
-0.008904 \\
0.002767\end{array}$ & $\begin{array}{r}0.0000: 28 \\
-0.0000017 \\
0.0000002\end{array}$ & $\mid \begin{array}{r}-0.0001391 \\
0.0000220 \\
-0.0000035\end{array}$ & $\begin{array}{c}0.001510 \\
-0.0002835 \\
0.0000513\end{array}$ & $\mid$ & $3-0.0000029$ & 0.0000319 \\
\hline
\end{tabular}

Table 3.

\begin{tabular}{c|c|c|c|c}
\hline$n$ & \multicolumn{2}{|c}{ Prolate } & \multicolumn{2}{c}{ Oblate } \\
\cline { 2 - 5 } & $D_{n}$ & $B_{n}$ & $i D_{n}$ & $i B_{n}$ \\
\hline 0 & -0.071692 & 0 & 0.270552 & 0 \\
2 & 0.025573 & 0.347777 & 0.061641 & -2.896763 \\
4 & 0.001183 & 0.633477 & 0.021645 & -5.255551 \\
6 & 0.000312 & 0.931033 & 0.007635 & -7.606669 \\
8 & 0 & 1.228769 & 0.003799 & -9.964251 \\
0 & 0 & 1.538722 & 0.002035 & -12.315873 \\
12 & 0 & 1.836458 & 0.001162 & -2.665964 \\
14 & 0 & 2.134194 & 0 & -16.991761 \\
16 & 0 & 2.431930 & 0 & -19.212287 \\
18 & 0 & 2.729667 & 0 & \\
\hline
\end{tabular}


Table 4. Prolate

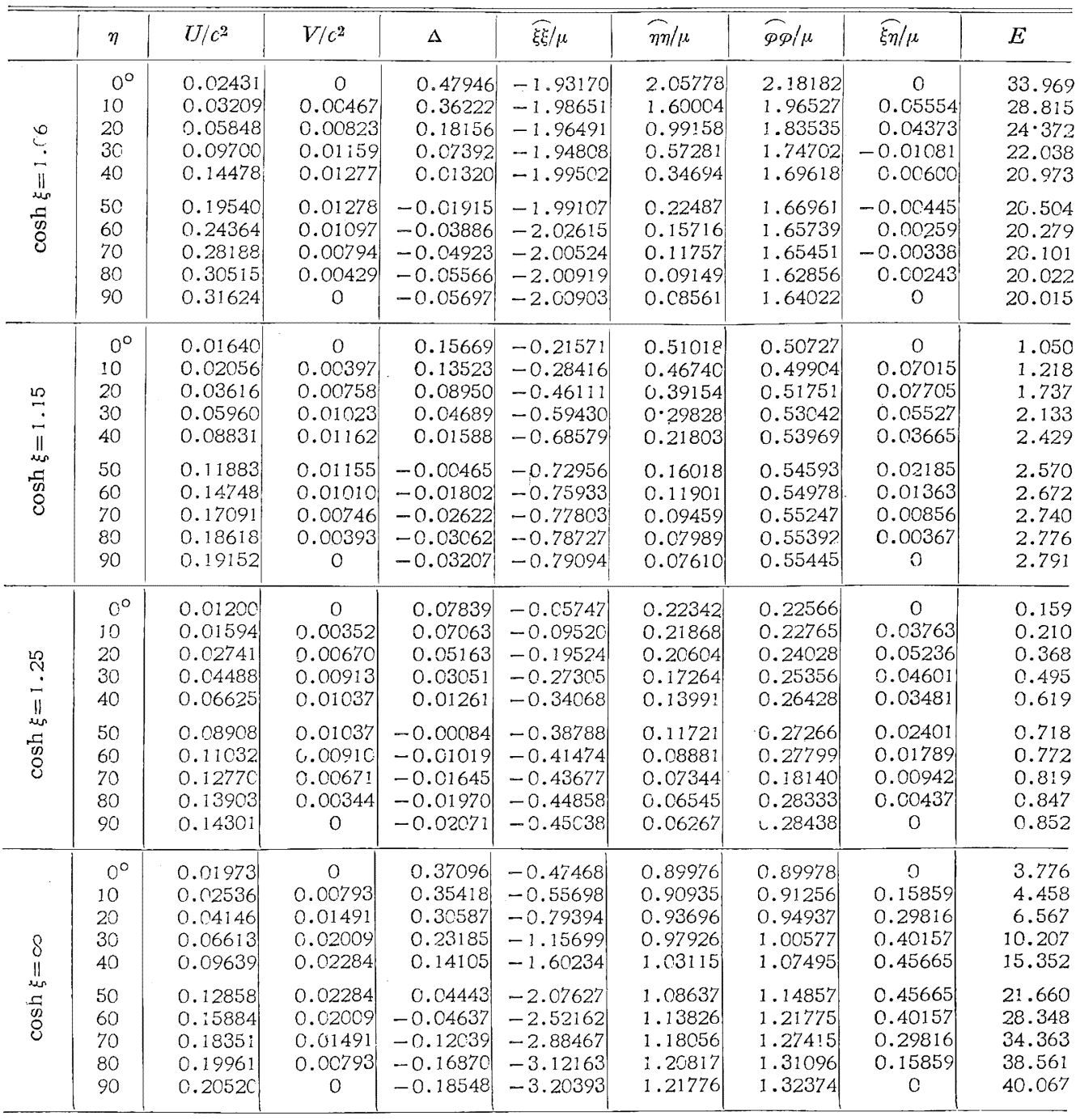

Table 5. Oblate

\begin{tabular}{|c|c|c|c|c|c|c|c|c|c|}
\hline & $\eta$ & $U / c^{2}$ & $V / c^{2}$ & $\Delta$ & $\widehat{\xi \xi} / \mu$ & $\widehat{\eta \eta} / \mu$ & $\widehat{\phi \varphi} / \mu$ & $\widehat{\xi \eta} / \mu$ & $E$ \\
\hline \multirow{10}{*}{ 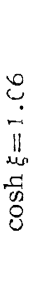 } & $0^{\circ}$ & 0.85986 & 0 & -0.54287 & -2.06468 & -0.34200 & -0.34203 & 0 & 5.498 \\
\hline & 10 & 0.83101 & -0.02844 & -0.53318 & -1.98684 & -0.33421 & -0.32406 & 0.00843 & 5.584 \\
\hline & 20 & 0.76356 & -0.05477 & -0.51009 & -2.03174 & $-0.2554 \mathrm{C}$ & -0.30590 & 0.03570 & 5.916 \\
\hline & 30 & 0.65500 & -0.07232 & -0.48180 & -2.00208 & $-0.1188 \mathrm{C}$ & -0.27122 & -0.05514 & 6.552 \\
\hline & 40 & 0.51829 & -0.08460 & -0.42529 & -2.00560 & 0.10341 & -0.22518 & 0.04831 & 7.683 \\
\hline & 50 & 0.37595 & -0.08276 & -0.33993 & -2.01045 & 0.44186 & -0.13407 & 0.08758 & 9.776 \\
\hline & 60 & 0.24140 & -0.07557 & -0.16677 & -1.91404 & 1.05363 & 0.02825 & 0.10953 & 14.488 \\
\hline & 70 & 0.13555 & -0.05730 & 0.12493 & -2.14783 & 1.87636 & 0.32528 & 0.11389 & 22.837 \\
\hline & 80 & 0.06018 & -0.03035 & 0.61904 & -2.14011 & 4.41884 & 0.80909 & 0.21229 & 62.124 \\
\hline & 90 & 0.03935 & 0 & 0.93051 & -2.51350 & 6.03342 & 1.14167 & 0 & 98.340 \\
\hline
\end{tabular}




\begin{tabular}{|c|c|c|c|c|c|c|c|c|c|}
\hline & $\eta$ & $J / c^{2}$ & $V / c^{2}$ & $\Delta$ & $\widehat{\xi \xi} / \mu$ & $\widehat{\eta \eta} / \mu$ & $\widehat{\rho \varphi} / \mu$ & $\widehat{\xi \eta} / \mu$ & $E$ \\
\hline $\begin{array}{l}\dddot{1} \\
11 \\
\text { wn } \\
0 \\
0\end{array}$ & $\begin{array}{l}60 \\
70 \\
80 \\
90\end{array}$ & $\begin{array}{l}35051 \\
22427 \\
12197 \\
05424 \\
03184\end{array}$ & $\begin{array}{c}-0.08279 \\
-0.07240 \\
-0.05302 \\
-0.02820 \\
0\end{array}$ & $\begin{array}{r}-0.23684 \\
-0.08454 \\
0.14900 \\
0.44662 \\
.0 .60419\end{array}$ & $\mid \begin{array}{l}-1.74449 \\
-1.59941 \\
-1.38364 \\
-0.91725 \\
-0.66848\end{array}$ & $\begin{array}{l}0.57519 \\
1.56181 \\
1.78715 \\
2.51005 \\
2.93362\end{array}$ & $\begin{array}{l}0.00884 \\
0.13994 \\
0.34176 \\
0.59338 \\
0.73051\end{array}$ & $\begin{array}{c}-0.10657 \\
-0.13852 \\
-0.26314 \\
-0.23560 \\
0\end{array}$ & $\begin{array}{r}8.84 \\
11.07 \\
15.53 \\
18.03 \\
19.78\end{array}$ \\
\hline 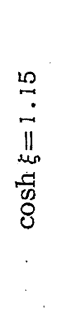 & $\begin{array}{l}0^{\circ} \\
10 \\
20 \\
30 \\
40 \\
50 \\
60 \\
70 \\
80 \\
90\end{array}$ & $\begin{array}{l}0.75226 \\
0.73022 \\
0.66738 \\
0.57097 \\
0.45254 \\
0.32648 \\
0.20915 \\
0.11462 \\
0.04814 \\
0.02642\end{array}$ & $\begin{array}{c}0 \\
-0.02809 \\
-0.05283 \\
-0.07098 \\
-0.08099 \\
-0.08265 \\
-0.07158 \\
-0.05324 \\
-0.02843 \\
0\end{array}$ & $\begin{array}{l}-0.36183 \\
-0.35549 \\
-0.33755 \\
-0.30414 \\
-0.24940 \\
-0.16454 \\
-0.03680 \\
.0 .13751 \\
0.32321 \\
0.41065\end{array}$ & $\begin{array}{l}-1.71748 \\
-1.70561 \\
-1.68822 \\
-1.64836 \\
-1.53147 \\
-1.45284 \\
-1.25200 \\
-0.92507 \\
-0.44190 \\
-0.21738\end{array}$ & $\begin{array}{r}-0.04533 \\
-0.02866 \\
0.03170 \\
0.13372 \\
0.30566 \\
0.54887 \\
0.89050 \\
1.31277 \\
1.62142 \\
1.78280\end{array}$ & $\begin{array}{r}-0.04533 \\
-0.04398 \\
-0.03161 \\
-0.00760 \\
0.02982 \\
0.08657 \\
0.17962 \\
0.30789 \\
0.42960 \\
0.49158\end{array}$ & $\begin{array}{c}0 \\
-0.01517 \\
-0.02701 \\
-0.05538 \\
-0.08690 \\
-0.13017 \\
-0.21247 \\
-0.30038 \\
-0.23967 \\
0\end{array}$ & $\begin{array}{l}5.592 \\
5.575 \\
5.711 \\
5.906 \\
6.276 \\
6.692 \\
7.416 \\
8.079 \\
6.782 \\
6.171\end{array}$ \\
\hline 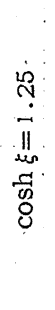 & $\begin{array}{l}0^{\circ} \\
10 \\
20 \\
30 \\
40 \\
50 \\
60 \\
70 \\
80 \\
90\end{array}$ & $\begin{array}{l}0.67398 \\
0.65411 \\
0.59776 \\
C .51093 \\
0.4 C 427 \\
0.29081 \\
0.18283 \\
0.09705 \\
0.04015 \\
0.02041\end{array}$ & $\begin{array}{c}0 \\
-0.02626 \\
-0.04928 \\
-0.06636 \\
-0.07545 \\
-0.07561 \\
-0.06658 \\
-0.04946 \\
-0.02634 \\
0\end{array}$ & $\begin{array}{r}-0.25850 \\
-0.25305 \\
-0.23623 \\
-0.206 C 6 \\
-0.15935 \\
-0.09229 \\
-0.00282 \\
0.10213 \\
0.19624 \\
0.23574\end{array}$ & $\begin{array}{r}-1.41905 \\
-1.40742 \\
-1.37643 \\
-1.31548 \\
-1.21408 \\
-1.05269 \\
-0.86644 \\
-0.43652 \\
-0.15334 \\
-0.00543\end{array}$ & $\begin{array}{l}0.06419 \\
0.07755 \\
0.12266 \\
0.21468 \\
0.31121 \\
0.45605 \\
0.61736 \\
0.77385 \\
0.87 C 83 \\
0.90497\end{array}$ & $\begin{array}{l}0.06416 \\
0 . c 6482 \\
0 . c 7267 \\
0.08593 \\
0.10627 \\
0.13535 \\
0.16977 \\
0.22054 \\
0.26188 \\
0.27930\end{array}$ & $\begin{array}{c}c \\
-0.01917 \\
-0.04692 \\
-0.07525 \\
-0.11638 \\
-0.16495 \\
-0.23134 \\
-0.24230 \\
-0.17361 \\
0\end{array}$ & $\begin{array}{l}4.400 \\
4.375 \\
4.363 \\
4.356 \\
4.193 \\
3.954 \\
3.602 \\
2.747 \\
1.773 \\
1.301\end{array}$ \\
\hline 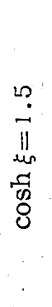 & $\begin{array}{l}00 \\
10 \\
20 \\
30 \\
40 \\
50 \\
60 \\
70 \\
80 \\
90\end{array}$ & $\begin{array}{l}0.55039 \\
0.53287 \\
0.48535 \\
0.4 .1214 \\
0.32356 \\
0.23076 \\
0.144 .74 \\
0.67598 \\
0.03139 \\
0.01595\end{array}$ & $\begin{array}{c}0 \\
-c .02192 \\
-0.04123 \\
-0.05557 \\
-0.06226 \\
-0.06322 \\
-0.05561 \\
-0.04121 \\
-0.02197 \\
0\end{array}$ & $\begin{array}{r}-0.13460 \\
-0.13016 \\
-0.11838 \\
-0.09846 \\
-0.06995 \\
-0.03334 \\
0.00887 \\
0.05085 \\
0.08300 \\
0.09519\end{array}$ & $\begin{array}{r}-0.89916 \\
-0.88571 \\
-0.84596 \\
-0.77454 \\
-0.65476 \\
-0.52946 \\
-0.35627 \\
-0.17441 \\
-0 . c 2755 \\
c .02957\end{array}$ & $\begin{array}{l}0.11673 \\
0.12314 \\
0.14187 \\
0.17088 \\
0.20920 \\
0.25668 \\
0.28879 \\
0.31694 \\
0.33055 \\
0.33501\end{array}$ & $\begin{array}{l}0.10989 \\
0.11568 \\
0.11460 \\
0.11248 \\
0.11185 \\
0.10970 \\
0.10990 \\
0.11176 \\
0.11351 \\
0.11421\end{array}$ & $\begin{array}{c}0 \\
-0.03081 \\
-0.06038 \\
-0.08723 \\
-0.10110 \\
-0.12685 \\
-0.12972 \\
-0.11187 \\
-0.05142 \\
0\end{array}$ & $\begin{array}{l}2.050 \\
2.026 \\
1.921 \\
1.730 \\
1.405 \\
1.134 \\
0.766 \\
0.441 \\
0.211 \\
0.149\end{array}$ \\
\hline 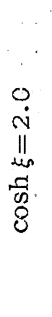 & $\begin{array}{l}0^{\circ} \\
10 \\
20 \\
30 \\
40 \\
50 \\
60 \\
70 \\
80 \\
90\end{array}$ & $\begin{array}{l}0.40054 \\
0.38862 \\
0.35470 \\
0.30253 \\
0.23856 \\
0.17 C 42 \\
0.10739 \\
0.05416 \\
0.020 c 6 \\
0.00821\end{array}$ & $\begin{array}{c}0 \\
-0.01659 \\
-0.03115 \\
-0.04198 \\
-0.04773 \\
-0.04773 \\
-0.04197 \\
-0.03117 \\
-0.01659 \\
0\end{array}$ & $\begin{array}{r}-0.05244 \\
-0.05094 \\
-0.04491 \\
-0.03575 \\
-0.02359 \\
-0.00937 \\
0.00540 \\
0.01859 \\
0.02783 \\
0.03116\end{array}$ & $\mid \begin{array}{r}-0.40322 \\
-0.39881 \\
-0.37224 \\
-0.32884 \\
-0.26967 \\
-0.19830 \\
-0.11507 \\
-0.05127 \\
-0.00068 \\
0.01779\end{array}$ & $\begin{array}{l}0 . c 725 c \\
0.07366 \\
0.07849 \\
0.08485 \\
0.09185 \\
0.09781 \\
0.10222 \\
0.1028 c \\
0.10235 \\
0.1021 c\end{array}$ & $\begin{array}{l}0.07336 \\
0.07280 \\
0.06921 \\
0.06522 \\
0.05989 \\
0.05364 \\
0.04772 \\
0.04141 \\
0.03736 \\
0.03590\end{array}$ & $\begin{array}{c}0 \\
-0.01440 \\
-0.02849 \\
-0.04150 \\
-0.05215 \\
-0.05772 \\
-0.05703 \\
-0.04648 \\
-0.02631 \\
0\end{array}$ & $\begin{array}{l}0.17 \\
0.09 \\
0.04 \\
0.02 \\
0.01\end{array}$ \\
\hline $\begin{array}{l}8 \\
\text { in } \\
\text { in } \\
\text { 1 } \\
0 \\
8\end{array}$ & $\begin{array}{l}0^{\circ} \\
10 \\
20 \\
30 \\
40 \\
50 \\
60 \\
70 \\
80 \\
90\end{array}$ & $\begin{array}{l}1.57168 \\
1.52449 \\
1.39035 \\
1.18494 \\
1.03273 \\
0.66447 \\
0.41236 \\
0.20685 \\
0.07271 \\
0.02612\end{array}$ & $\begin{array}{c}0 \\
-0.06605 \\
-0.12413 \\
-0.16725 \\
-0.19019 \\
-0.19019 \\
-0.16725 \\
-0.12413 \\
-0 . c 6625 \\
0\end{array}$ & $\begin{array}{r}-3.48988 \\
-2.95012 \\
-2.54771 \\
-1.93118 \\
-1.17487 \\
-0.37007 \\
.0 .38624 \\
1.00276 \\
1.40518 \\
1.54494\end{array}$ & $\mid \begin{array}{r}-28.2271 \\
-27.34195 \\
-24.79331 \\
-20.88856 \\
-16.09855 \\
-11.00149 \\
-6.21148 \\
-2.36673 \\
0.24191 \\
1.12706\end{array}$ & $\begin{array}{r}5.32602 \\
5.17156 \\
5.07074 \\
5.03573\end{array}$ & \begin{tabular}{r|}
6.38891 \\
$6.249 c 5$ \\
5.84663 \\
$5.23 c c 8$ \\
4.47375 \\
3.66894 \\
2.91261 \\
$2.296 C 6$ \\
1.89364 \\
1.75388
\end{tabular} & $\begin{array}{c}0 \\
-1.32101 \\
-2.48266 \\
-3.34489 \\
-3.80367 \\
-3.80367 \\
-3.34489 \\
-2.48266 \\
-1.32101 \\
0\end{array}$ & $\begin{array}{r}2362.74 \\
2261.27 \\
1926.59 \\
1467.81 \\
987.40 \\
578.24 \\
289.31 \\
122.37 \\
46.60 \\
26.44\end{array}$ \\
\hline
\end{tabular}

In tables 4 and 5 , when cosh $\xi=\infty$, unc of eacn column is as follows:

$U / c^{2}, V / c^{2} \cdots \cdots e^{-\xi} \Delta, \widehat{\xi \xi} / \mu, \widehat{\eta \eta} / \mu, \widehat{\phi \phi} / \mu, \widehat{\xi \eta} / \mu \cdots \cdots e^{-3 \xi} \cdot E \cdots \cdots e^{-6 \xi}$ 